\title{
6 Unsichtbarer Einfluss: Wirtschaftsberater in Sansibar und Dar es Salaam
}

Nach der Einführung in das Feld der Entwicklungspolitik mit seinen wechselhaften Rahmenbedingungen sowie einem Blick auf Akteure und ihre Motive und Handlungsorientierungen stehen in den folgenden drei Kapiteln Interaktionen zwischen expatriates und Counterparts in konkreten Arenen im Vordergrund. ${ }^{1}$ In diesen Arenen lässt sich eine gemeinsame Entwicklungslinie identifizieren. Während die 1960er und frühen 1970er-Jahre breite Gestaltungsmöglichkeiten für eine Reihe von Akteuren boten, mussten die meisten Beteiligten - zumindest all jene ohne privilegierten Zugang zu ökonomischem Kapital - in den 1980er-Jahren auf engem Raum manövrieren. Politische Visionen mit langfristigem und oft globalem Horizont verloren an Praxisrelevanz und wurden von Strategien zur kurzfristigen Krisenbearbeitung im nationalen oder lokalen Rahmen verdrängt. Diese Verschiebungen werden in der diachronen Perspektive der Fallstudien deutlich. Während westdeutsches Entwicklungspersonal von den materiellen Knappheiten persönlich viel weniger betroffen war, mussten sie ihre Handlungsweise doch auf diese neuen Voraussetzungen einstellen - wie die Fallstudien zur Universität Dar es Salaam und dem regionalen Entwicklungsprogramm TIRDEP zeigen. Den Beginn macht jedoch ein Einblick in die Tätigkeit deutscher Finanz- und Wirtschaftsberater in Tansania.

Eine beratende Funktion bot im Gegensatz zu einer ausführenden Tätigkeit eine Reihe von Vorteilen. Sie erlaubte Distanz zu Alltagsaufgaben und Übersicht, ermöglichte eher den Zugang zu EntscheidungsträgerInnen und die Verfolgung politischer Zielsetzungen durch die Erarbeitung von Konzepten und Vorlagen. Ein Berater galt als erfolgreich, wenn er das Vertrauen des jeweiligen Counterparts gewonnen hatte und seine Ratschläge, die sowohl der politischen Ausrichtung des Gastlandes wie auch den wirtschaftlichen und politischen Interessen des Entsendelandes zu entsprechen hatten, Beachtung fanden. Beratungsaufgaben waren aber auch besonders heikel, weil sie noch mehr als andere Einsatzmodi von Counterpartinteressen und politischen Konjunkturen abhingen. Die goldene Regel aus der Erfahrung der beratenden Entwicklungsarbeit lautete daher: „Die

$1 \mathrm{Zu}$ Arenen der Entwicklungsarbeit siehe Olivier de Sardan, Anthropology and Development, S. 185-190; Kate Crehan/Achim von Oppen, „Understandings of Development“: An Arena of Struggle: The Story of a Development Project in Zambia, in: Sociologia Ruralis 28/2-3 (1988), S. $113-145$.

Ә OpenAccess. ( 2021 Eric Burton, publiziert von De Gruyter. (cc)BY Dieses Werk ist lizenziert unter der Creative Commons Attribution 4.0 International Lizenz. https://doi.org/10.1515/9783110705621-009 
Berater müssen sich ihre Einflussmöglichkeiten erst mühsam verschaffen. “2 In manchen Fällen war Erfolg oder Misserfolg auf das Geschick des Beraters ${ }^{3}$ oder die persönliche Einstellung des jeweiligen Counterparts zurückzuführen, aber selbst in diesen Fällen müssen Erklärungen für eine fruchtbare, angespannte oder schlicht inexistente Kooperation mit Counterparts auch auf anderen Ebenen gesucht werden. Vier Variablen hatten signifikanten Einfluss auf die Beratungstätigkeit: die historisch spezifische Konstellation (d.h. politische und wirtschaftliche Rahmenbedingungen); das direkte institutionelle Umfeld und die Aufgabenstellung des Beraters; die Interessen des bzw. der jeweiligen Counterparts - sofern ein solcher, was nicht selbstverständlich ist, überhaupt vorhanden war - und die Möglichkeit, strategische Allianzen mit ihnen einzugehen; sowie schließlich der Habitus und die persönlichen Ressourcen des jeweiligen Beraters, was sowohl das Rollenverständnis wie auch fachliche und sprachliche Kenntnisse bis hin zu Umgangsformen umfasst.

Die allgemeinen Anforderungen an Berater in der Entwicklungspolitik unterschieden sich nicht wesentlich von den Qualitäten, die Beratungspersonen von Königen und Herrscherinnen von jeher aufweisen mussten: mit den Mächtigen, die beraten werden sollten, vorsichtig umzugehen, heikle Themen zu meiden und Maßnahmen einzuflüstern statt einzufordern. Ein entscheidender Unterschied zu historischen Vorformen ist jedoch, dass die Regierungsberater des Entwicklungszeitalters an einen doppelten Handlungsrahmen gebunden waren: Auch das Entsendeland hatte ein Interesse an dem Einsatz und eine Entfernung von diesen Interessen konnte schwere Konsequenzen für die individuelle Biografie nach sich ziehen. Zweitens bestand bei Beratern in der Entwicklungsarbeit oft der innere Drang oder der äußere Druck, Dinge selbst in die Hand zu nehmen und auszuführen - mit dem Risiko, dass Misserfolge dann in ihre Verantwortung fielen.

Die vier Mikrostudien in diesem Kapitel beleuchten verschiedene Beraterpositionen und Kontexte in chronologischer Anordnung. Den Beginn macht eine Betrachtung der Tätigkeit von DDR-Finanzberatern im postrevolutionären Sansibar. Es folgt der Blick auf zwei westdeutsche Beratungsprojekte in der tansanischen Wirtschaft zwischen 1970 und 1981; den Abschluss macht eine Analyse der Tätigkeit von DDR-Planungsberatern auf dem Festland in den 1980er-Jahren. Die Studien zeigen, wie sehr die Berater auf ihr Handlungsumfeld angewiesen waren, wie schnell sich die äußeren Bedingungen ändern konnten und welche Strategien

2 BArch Koblenz, B 213/33088, Metschies (GTZ), Stellungnahme des Projektsprechers zu den Berichten Nr. 1-3 des Beratungsprojektes NTC Tanzania, o.O., o.D., S. 4. Zu Beispielen aus anderen Beraterkontexten siehe auch Kap. 1.

3 Im Quellenmaterial scheinen ausschließlich Männer auf, weswegen in diesem Kapitel die männliche Form beibehalten wird. 
sie nutzten, um ihren Einfluss zu vergrößern; aber auch, welche Methoden Counterparts anwandten, um die Berater in die Verfolgung ihrer Interessen einzubinden, sie zu ignorieren oder aktiv zu marginalisieren. Außerdem verdeutlicht der Blick in die Aushandlungen auch, wie die ephemeren Ziele „Dekolonisierung“ und „Entwicklung“ erreicht werden sollten - und was sie in konkreten Kontexten in verschiedenen Perspektiven bedeuteten.

\title{
6.1 Antiimperialistische Sündenböcke: DDR-Finanzberater im postrevolutionären Sansibar (1964-1970)
}

Auf Sansibar genossen ostdeutsche Berater einen Einfluss und ein Ansehen wie selten zuvor und danach in der Geschichte der „sozialistischen Hilfe“ der DDR. „Sansibar erblühte“, schrieb die Regierungszeitung Kweupe in einem Bericht über die Verabschiedung des ersten DDR-Finanzberaters Martin Gentsch im Januar 1967, drei Jahre nach der Revolution. Sansibars Präsident (und gleichzeitig Tansanias Vizepräsident) Abeid Amani Karume sah Sansibar zu großem Dank verpflichtet, Gentsch sei durch seine Leistungen zu einem Tansanier geworden. ${ }^{4}$ Es blieb allerdings ein zeitlich scharf begrenzter Einfluss. 1970, in einer Rede vor 150 sansibarischen FunktionärInnen, allen Konsuln sowie einigen Gästen vom Festland holte Karume zum Schlag gegen die DDR-Berater aus:

\begin{abstract}
Wir sind zwar seit 1964 politisch frei, aber wir können noch nicht sagen, daß wir ökonomisch frei sind. In der Politik haben wir ständig Erfolge erreicht, aber in der Ökonomie haben uns die ausländischen Experten alles verdorben. [...] Die ausländischen Ökonomie- und Finanzexperten haben unsere Wirtschaft nahe an den Bankrott gebracht. Ökonomie- und Finanzexperte bei uns kann nur sein, der jedem Bürger zu essen und zu trinken sowie eine Schlafstätte verschafft. ${ }^{5}$
\end{abstract}

Anstelle der ausländischen Experten und der Sansibaris mit ausländischen Hochschulabschlüssen sollten fortan zwei loyale sansibarische Funktionäre die Wirtschaft in die Hand nehmen. Beide, wie Karume unumwunden zugab, „haben keine Hochschulbildung. Sie sind keine Ökonomen. [...] Aber sie sind politische Führer und werden die Ökonomie richtig leiten." ${ }^{\text {" }} \mathrm{Zu}$ diesem Zeitpunkt negierte Karume die Bedeutung von Expertenwissen und höherer Bildung. Die Devalua-

4 BArch Berlin, DN 1/15578, Kweupe, Der 1. Vizepräsident verabschiedete Herrn Gentsch (Übersetzung), 1.2.1967

5 BArch Berlin, DC 20/11525, DDR-Konsulat Sansibar, Zusammenfassende Nachschrift der Rede des 1. Vizepräsidenten der VRT, A.A. Karume, am 30.5.1970, Sansibar, 2.6.1970, Bl. 74, 80

6 Ebd., Bl. 75 
tion von Fachwissen wurde auch in andere Bereiche übertragen. „Wir brauchen in Sansibar keine Ingenieure“, setzte Karume fort: „Wir bauen unsere Häuser selbst. Ich selbst, wie ihr alle wisst, baue Häuser ohne Ingenieur zu sein. “7 Karumes Zuhörerschaft konnte kaum verborgen bleiben, dass er mit seinen Aussagen nicht etwa das chinesische Personal, sondern die Finanzberater, Planungsexperten, Baufachleute und Architekten aus der DDR attackierte.

Die Rede hatte in erster Linie innenpolitische Motive, denn mit ihr verkündete Karume die Ersetzung aller Ministerposten durch Vorsitzende im Revolutionsrat, was ihm weitere Befugnisse gab und seine Machtposition gegen einflussreiche Kritiker, von denen viele gegen eine Anbindung an die Sowjetunion und die DDR waren, stärkte. Die Ursachen für den Bruch zwischen Karume und den DDR-Beratern und -Experten liegen freilich tiefer und sind nicht nur in Meinungsverschiedenheiten zu suchen. Wie bereits gezeigt, endeten die kommerziellen DDRProjekte fast ausnahmslos in Misserfolgen (siehe Kapitel 3). In Karumes Rede spiegelten sich aber auch Spannungen, die bereits in den Jahren zuvor immer wieder aufgetreten waren. Karume betonte das Primat politischer und sozialer Kriterien vor (volks-)wirtschaftlichen und fachlichen Gesichtspunkten und verfolgte damit die Nationalisierung und Konzentration der Macht in seinen Händen. Innenpolitische Machtkämpfe hatten auch dem Finanzminister Twala, dem wichtigsten Counterpart der DDR-Finanzberater, der von Anfang an ein wichtiger Verbündeter gewesen war, 1967 das Leben gekostet.

\section{Die Dekolonisierung der Wirtschaft (1964-1967)}

Etwas mehr als zwei Monate nach der Revolution am 18.3.1964 trat Martin Gentsch, ein erfahrener Beamter aus dem DDR-Finanzministerium, seine Position als Finanzberater der sansibarischen Regierung an. Nur eine Woche nach seiner Ankunft und damit deutlich früher als geplant wurden die letzten britischen Beamten, die nach der Revolution noch im Ministerium verblieben waren, aus

7 Ebd., DDR-Konsulat Sansibar, Zusammenfassende Nachschrift der Rede des 1. Vizepräsidenten der VRT, A.A. Karume, am 30.5.1970, Sansibar, 2.6.1970, Bl. 77. Tatsächlich sahen sich DDR-Architekten und Mitglieder von Freundschaftsbrigaden immer wieder „in Oppositionsstellung zu Karume gezwungen“, wenn dieser persönlich auf den Baustellen erschien und Änderungen forderte, die aus Sicht der DDR-Kader völlig unpassend waren oder internationale Standards missachteten. BArch Berlin, DC 20/11525, DDR-Konsulat an MfAA, Sansibar, 28.4.1970, Bl. 239. Die Meinungsverschiedenheiten und Aushandlungsprozesse in den Bauprojekten werden analysiert in Ludger Wimmelbücker, Architecture and City Planning Projects of the German Democratic Republic in Zanzibar, in: The Journal of Architecture 17/3 (2012), S. 407-432. 
dem Dienst entlassen. Dank des Einflusses linker Kräfte auf den Inseln mussten die Vorstellungen einer sozialistischen Moderne keinesfalls von ostdeutschen (oder chinesischen) Akteuren „importiert“ werden: Sie hatten bereits Wurzeln geschlagen. Gentsch traf auf weit gereiste sansibarische Marxisten, die einflussreiche Positionen im Revolutionsrat und Ministerposten auf dem Festland besetzten. Zwölf Tage nach seiner Ankunft wurde Abdulaziz Twala zum Finanzminister ernannt, der zur sowjetfreundlichen Fraktion gehörte und integraler Teil des „linken“ Flügels in der stark fraktionierten sansibarischen Politiklandschaft war. Schon vor der Unabhängigkeit 1963 und der Revolution im Januar 1964 hatte sich die Parteilandschaft weiter fragmentiert, wobei einige Marxisten, die zuvor aus Karumes ASP ausgetreten waren, später im Revolutionsrat vertreten waren. Twala kam aus der Gewerkschaftsbewegung, war überzeugter Marxist und stolz auf seine Ausbildung in Moskau, die er noch vor der Unabhängigkeit abgeschlossen hatte. ${ }^{8}$ Die sansibarische Verfassung wollte er nach sowjetischem Vorbild gestalten, da hier wie dort die Macht der Regierung dem revolutionären Umsturz entsprungen war. ${ }^{9}$

Twala bereitete Gentsch den bestmöglichen Empfang, stellte ihm einen Regierungswagen zur Verfügung und ließ ihn samt mitreisender Ehefrau im Nachbarhaus einziehen, um einen stetigen Austausch zu ermöglichen. Gentsch bekam das neue, stark reduzierte Höchstgehalt für Ausländer, 100 britische Pfund in Devisen. Das Vertrauensverhältnis war sofort hergestellt. Twala versorgte Gentsch regelmäßig mit den neuesten Informationen und Dokumenten aus dem Kabinett und gab Order an seine Untergebenen, „dass während seiner Abwesenheit vom Ministerium meine Vorschläge als seine Weisungen gelten. Bisher wurde in allen Fällen entsprechend meinen Vorschlägen verfahren. "10 So übernahm Gentsch von Anfang an eine Rolle, die über eine Beraterfunktion hinausging. Twalas Vision vom sansibarischen Finanzsektor basierte auf der Vorstellung einer gemeinsamen sozialistischen Moderne, in der Sansibar von der DDR lernen konnte. Folgerichtig unterstützte er die Ausbildung von Sansibaris in der DDR. Er beschrieb es als „our desire that some of our best and experienced officers should have the chance to visit the G.D.R. in order to get acquainted with the socialist system in the field in which they are working in Zanzibar“. ${ }^{11}$

8 Donald Petterson, Revolution in Zanzibar. An American's Cold War Tale, Boulder 2002, S. 142. 9 Burgess, A Socialist Diaspora, 278. Zu Twalas Auseinandersetzungen mit seinem FestlandCounterpart Amir Jamal um die Gründung einer sansibarischen Nationalbank siehe Issa G. Shivji, Pan-Africanism or Pragmatism? Lessons of the Tanganyika-Zanzibar Union, Dar es Salaam 2008, S. $133-141$.

10 BArch Berlin, DN 1/15578, Martin Gentsch, 2. Bericht, Sansibar, 11.4.1964.

11 BArch Berlin, DN 1/15579, Abdulaziz A. K. Twala an Min. der Finanzen, Sansibar, 8.10.1965. 
Zwar hatte Gentsch, nach Absprache mit dem DDR-Konsulat, nur einen Beraterposten und nicht die Position des Staatssekretärs übernommen, aber trotzdem drehte sich sein Alltag anfangs um exekutive Aufgaben. Nachdem Gentsch einen Überblick über die Finanzlage und die Alltagsgeschäfte gewonnen hatte, trat die Frage der Dekolonisierung in den Vordergrund, da, wie er erkannte, die Finanzpolitik bisher in London gemacht worden war und Sansibar sich im Zustand „völliger Abhängigkeit“ befand. Als übergeordnetes Ziel sah Gentsch, Sansibars „Abhängigkeit vom Imperialismus durch Stärkung der politischen und ökonomischen Macht des Landes zu vermindern und zu beseitigen“. ${ }^{12}$ Ein erster Schritt war die Ersetzung der kolonialen Gesetzgebung durch neue Verordnungen zum Staatshaushalt und zur Finanzrevision. Dazu gehörte zuerst der Grundsatz, dass der Haushalt immer einen Überschuss haben sollte, um Auslandsverschuldung zu vermeiden. Weiters wurden Entscheidungsbefugnisse von der Beamtenauf die politische Ebene verlagert und Luxusgüter, deren Import nur der „Bourgeoisie“ zugute kam, aber den Devisenhaushalt enorm belastete, mit höheren Steuersätzen belegt und ihrer Zollpräferenzen entledigt. Außerdem wurden, was Gentsch zufolge vor allem auf die „indisch[e] Bourgeoisie abzielte“, eine Handelssteuer für Importwaren eingeführt und viele Privilegien der Beamtenschaft hohe Pensionen, Wohnungs- und Urlaubszuschüsse, Kilometergeld ohne Nachweise - beseitigt. $^{13}$ Allein die Maßnahmen zum Abbau der Beamtenprivilegien senkten die staatlichen Ausgaben um ganze zehn Prozent. Welche dieser Maßnahmen von Gentsch vorgeschlagen wurden, lässt sich aus seinen Berichten nicht eruieren: Das „Ich“ taucht, seinem Credo (siehe unten) gemäß, dass alle Entscheidungen die Entscheidungen der Sansibaris seien, in diesem Maßnahmenkatalog nicht auf; deutlich wird nur, dass er in mehreren Fällen - und hier spielten immer wirtschaftliche Argumente die Hauptrolle - eine schrittweise statt einer radikalen Umsetzung empfahl.

Eine weitere Maßnahme zur Dekolonisierung bestand darin, in Großbritannien angelegte Wertpapiere aufzulösen und fortan keinerlei staatliche Aufträge mehr an die Crown Agents London, die britische Handelsagentur für die Kolonien, zu vergeben. Bis 1966 setzte Karume auf die Strategie, Sansibar vom kapitalistischen Wirtschaftskreislauf abzukoppeln und ökonomisch in die sozialistische Staatengemeinschaft zu integrieren, solange das für den Aufbau Sansibars notwendig schien. In Bezug auf Kredite war das relativ leicht machbar: Nach der

12 BArch Berlin, DN 1/15578, o.A. [Gentsch], Ergebnisse auf dem Finanzgebiet in Sansibar, o.O., o.D. [1967], S. 6.

13 Ebd., Gentsch, Veränderungen auf dem Finanzgebiet, die dazu beitragen, Sansibars Abhängigkeit vom imperialistischen Kapitalismus zu beseitigen und sein erneutes Eindringen zu verhindern, Sansibar, 18.8.1966. 
Revolution nahm Sansibar nur in kommunistischen Ländern Kredite auf, die aus politischen Gründen auch zu sehr günstigen Konditionen offeriert wurden. Schwieriger gestaltete sich die sofortige Abkopplung im Handel, aber auch im Produktionsbereich: Hier empfahlen DDR-Berater, für die Produktion von Dosenfrüchten Konserven von einer Firma in Dar es Salaam zu importieren und eine englische Anlage für die Reparatur von Fischkuttern zu nutzen. Karume sperrte sich dagegen, wie das DDR-Konsulat in einem Besprechungsprotokoll festhielt:

Die Imperialisten werden aber alles versuchen, um uns Schaden zuzufügen. Aus diesem Grunde dürfen wir uns in keinerlei Abhängigkeit von ihnen begeben, besonders dürfen wir das nicht auf dem Gebiet der Wirtschaft. Wir dürfen den Imperialisten auch nicht die kleinste Angriffsfläche bieten. Aus diesen Gründen ist es notwendig, die Konservenbüchsen selbst zu produzieren und eine eigene Reparaturbasis für die Fischkutter aufzubauen. ${ }^{14}$

Karume war in seiner antiimperialistischen Haltung jedoch wenig konsequent und ließ sich eher von identitätspolitischen Gesichtspunkten leiten. Nach weiteren Diskussionen segnete er den Konservenimport doch ab, weil es sich um eine englische Firma handelte - „Bezug von einer indischen Firma sei jedoch nicht möglich“, wie er präzisierte. ${ }^{15}$ Das MfS zitierte sansibarische Stimmen, die Karumes „spontane“ Wirtschaftspolitik beanstandeten und hielt fest, dass Entscheidungen wie das Importverbot für alle privaten Händler vom 17.11.1967 nicht mit Beratern aus den sozialistischen Ländern (sprich: DDR oder Sowjetunion) abgesprochen worden seien. ${ }^{16}$ Dass die Verstaatlichung des Einzelhandels zu eklatanten Versorgungsproblemen führte und die ,indische Kaufmannsschicht [...] offenbar liquidiert werden“ sollte, fiel unter jene Fragen, die die DDR-Finanzberater für politisch unantastbar erachteten. ${ }^{17}$ Womöglich leisteten sich DDR-Berater hier auch einen (unsichtbaren) Wettstreit mit China. Historiker Gregg Brazinsky zufolge unterstützten chinesische Wirtschaftsberater Karumes radikale Nationalisierungstendenzen und empfahlen ausdrücklich, den Kleinhandel in staatliche Hände zu legen. ${ }^{18}$ Einsehbar war für die DDR jedoch nur, was sansibarische Akteure forderten und umsetzten. Dabei kam es zu weiteren Unstimmigkeiten.

14 BArch Berlin, DE 1/52555, DDR-Konsulat, Aktenvermerk über eine Aussprache mit dem 1. Vizepräsidenten der VRT Karume am 6.5.1966, Sansibar, 12.5.1966, S. 3.

15 Ebd., S. 4.

16 BStU, MfS, HVA 229, Einzel-Information über außen- und innenpolitische Probleme Tansanias, Berlin, 15.12.1967, Bl. 135.

17 BArch Berlin, DN 1/15578, Nimmrich an MdF, Sansibar, 18.12.1967.

18 Gregg A. Brazinsky, Winning the Third World: Sino-American Rivalry during the Cold War 2017, $293-294$. 
Young-Sun Hong hat gezeigt, dass DDR-Pläne in Nordkorea auf Gegenwehr stießen, weil die DDR-Standards für lokale Verhältnisse zu kostspielig waren. ${ }^{19}$ In Sansibar war ebenfalls zu erwarten, dass z. B. die Instandhaltungskosten für die gigantischen Wohnbauprojekte den Staatshaushalt in Zukunft enorm belasten würden. Davon zeigten sich die Sansibaris allerdings wenig besorgt. Zentral war eher die Frage, in welche Sektoren der Staat investieren sollte. Karume bevorzugte aus politischen und Prestigegründen die Investition in „moderne“ Wohnhäuser, Bildung (die Schulgebühren wurden vollständig abgeschafft), Gesundheit und Sportstätten. Finanz- und Planungsberater der DDR wiesen wiederholt darauf hin, dass die Regierung möglichst in den produktiven Sektor und nicht nur in die physische und soziale Infrastruktur investieren sollte, da letztere weitere Kosten verursache (also einer „Konsumption“ von Kapital gleichkäme), aber weder die Produktionskapazitäten vergrößere noch Einkommen generiere. Zur Untermauerung dieser Sicht verwiesen sie - ein historisches anstelle eines theoretischen Arguments verwendend - auf die „Erfahrungen in der DDR und in anderen sozialistischen Ländern“.20 Die Bevorzugung des Produktionssektors entsprach auch dem zeitgenössischen Vorgehen in der DDR unter Walter Ulbricht; ebenso drängte eine sowjetische Beratergruppe Ghanas Präsident Kwame Nkrumah 1965, mehr Ressourcen in die Produktion und weniger in soziale Dienstleistungen zu investieren, um die ökonomische Basis für eine weitere Entwicklung zu schaffen. ${ }^{21}$ DDR-Finanzberater und die Planungsgruppe empfahlen daher, chinesische und ostdeutsche Rahmenkredite voll auszuschöpfen, um die Produktion zu steigern und die fast allein auf Nelken basierende Exportstruktur zu diversifizieren bzw. die Abhängigkeit von Importen und Exporterlösen zu verringern. Der DDRKonsul berichtete - in der klassischen rhetorischen Figur des Beratereinflusses, die den Meinungen der Counterparts wenig Beachtung zubilligt - dass „der Finanzminister Twala [...] aufgrund der Hinweise des Gen[ossen] Gentsch Karume überzeugen“ konnte, „die unökonomischen Objekte wie Stadionbau“ zu vertagen und angesichts der Überschusslage im Haushalt verstärkt in die Produktion zu investieren. $^{22}$

Die meisten staatlichen Industrieprojekte - Frucht- und Milchverarbeitung, Fischfang und -verarbeitung, Ölpressen - wurden an die DDR vergeben. Während manche Sansibaris das DDR-Engagement insgesamt argwöhnisch beäugten, differenzierten andere (wie Twala) zwischen den „Außenhändlern“ und der politi-

19 Hong, Cold War Germany, S. 51.

20 BArch Berlin, DE 1/52555, Leiter der Expertengruppe Klaus Thomas, Information zu einigen Grundproblemen der Planstudie, Sansibar, 31.3.1966, S. 2.

21 Willard S. Thompson, Ghana's Foreign Policy, 1957-1966, Princeton 1969, S. 401.

22 SAPMO BArch Berlin, DY 30/98144, DDR-Konsul an Kern, Sansibar, 23. 8.1966, Bl. 97. 
schen Vertretung der DDR: Sie schätzten die ostdeutsche „Hilfe“, aber warfen den „Außenhändlern“ Profitdenken vor. Womöglich auch aus diesem Grund - aufseiten der DDR vermutete man jedoch vor allem wachsenden chinesischen Einfluss - wurde der Vertrag mit dem DDR-Berater für Außenhandelsfragen schon im Juni 1965 aufgelöst. ${ }^{23}$ Die Finanzberater und Planungsexperten betonten in ihren Berichten ebenfalls immer wieder, dass die undurchdachten Angebote der DDRAußenhändler den Ruf der DDR schädigten und die alltägliche Arbeit dadurch erheblich erschwerten. Gentsch und die Planungsexperten echauffierten sich über die miserabel kalkulierten (zum Teil verfünffachten sich die Kosten von einem aufs nächste Angebot), wirtschaftlich nicht nachhaltigen (fehlende Absatzmärkte) und unzuverlässigen (manche Offerten zog die DDR zurück) Angebote der Außenhändler. Trotz seiner reservierten Haltung gegenüber dem DDR-Wirtschaftsflügel konnte Gentsch als DDR-Auslandskader Sansibar jedoch unmöglich empfehlen, die ostdeutschen Angebote abzulehnen.

\section{Gescheiterte Berater (1965-1967)}

Ein weiterer Grund für die Verschlechterung des Bildes, dass sich Sansibaris von DDR-Beratern machten, war die ungenügende Eignung oder Vorbereitung mancher Entsandter. Zwei Berater, die zwischen 1965 und 1966/67 dem neugebildeten staatlichen Baubetrieb auf die Beine helfen sollten, galten in den Augen des sansibarischen Ministeriums nicht einmal als Experten. Der Building Adviser wurde in einem Schreiben als „Bauarbeiter mit durchschnittlichem Wissen“ beschrieben, während der Economic and Financial Adviser lediglich das „Wissen eines Buchhalters“ vorweise. ${ }^{24}$ Das DDR-Ministerium, das die beiden Kader entsandt hatte, wies den Vorwurf der mangelhaften Qualifikation zurück (beide waren Diplom-Volkswirte und hatten Leitungsfunktionen in der DDR eingenommen), räumte aber ein, dass „persönliche Schwächen“ zur baldigen Abberufung des ersten und „Widersprüche mit neu zugeführten [sansibarischen] Leitkadern [...] im Zusammenhang mit dem Fehlen englischer Sprachkenntnisse“ zur Abberufung des zweiten Beraters geführt hatten. Die Schlussfolgerung, dass „ein wesentlicher Teil der Gründe für die geringe Effektivität [...] auf sansibarischer Seite“ lag, demonstriert, dass das Bauministerium Faktoren wie persönlicher Eignung, dem Umgang mit Counterparts und sogar grundlegendsten Sprachkenntnissen

23 BArch Berlin, DN 10/985, Ebert an Dietrich, Sansibar, 17.6.1965.

24 BArch Berlin, DC 20/11525, Hamdan Muhiddin (Ministerium für Arbeit, Verkehr und Energie) an DDR-Konsulat, „Unsere Beschwerden über Experten und Ausrüstungen aus Ostdeutschland“ (Übersetzung), Sansibar, 21.10.1968, Bl. 181. 
keine große Bedeutung beimaß. Beide Seiten führten zudem auf je eigene Weise ökonomische Argumente ins Feld. Während das sansibarische Ministerium die Gehälter und weiteren anfallenden Kosten für die beiden Experten auflistete und als exorbitant erachtete, wog das DDR-Ministerium das Gehalt mit (hypothetischen) „Einsparungen bei der Ausführung von Bauvorhaben [auf], die die Ausgaben der sansibarischen Seite für ihren Einsatz überschreiten““.25 Dieselbe Argumentationsweise tauchte auch in Aussagen von BRD-Beratern auf (siehe unten) und war Kennzeichen einer ökonomistischen Rationalität, in der politische Fragen nicht offen thematisiert wurden.

Im April 1967 traf endlich der Bankberater ein, um den Gentsch schon 1964 gebeten hatte. Der Nachfolger von Gentsch, Hans Nimmrich, empfahl jedoch, den Neuankömmling umgehend wieder abzuberufen und einen Ersatzmann „mit besseren Sprachkenntnissen und etwas mehr Kämpferherz" bereitzustellen. ${ }^{26}$ Tatsächlich musste Bankberater Klee schon im Oktober wieder in die DDR zurückkehren, weil sich Twala nach einer Verlängerung der Einarbeitungsfrist dazu entschieden hatte, keinen Dienstvertrag anzubieten. Klee sah sein vollständiges Scheitern nicht in seiner Person, sondern in den politischen und wirtschaftlichen Rahmenbedingungen begründet: eine zunehmend starke Gruppierung in Regierung und Revolutionsrat, die gegen ausländische Berater sei; die Union mit dem Festland, die eine Reduzierung von Beratern aus der DDR und der Sowjetunion nach sich ziehe, aber auch die DDR-,,Investruinen“ führte er als Ursachen für sein Scheitern an. ${ }^{27}$ Ein weiterer externer Faktor war, wie Klee vermutete, der Einfluss Chinas. Neben Investitionen in eine Schuhfabrik und Karumes Prestigeprojekt Stadionbau stellte China der neu gegründeten sansibarischen Volksbank ein Grundkapital von 800.000 britischen Pfund zur Verfügung, vergab Konsumkredite und übte womöglich auch Druck auf Sansibar aus, DDR-Beratern keinen Einblick in die chinesisch-sansibarischen Finanzbeziehungen zu geben.

Die Hauptgründe, die Sansibar gegenüber der DDR angab, um die Abberufung der Berater zu rechtfertigen - mangelhafte Englischkenntnisse sowie fehlende Notwendigkeit eines Bankberaters - waren laut DDR-Konsulat zutreffend. Für einen chinesischen Einfluss hatte man keinerlei Hinweise. Die Unionsfrage mochte eine Rolle spielen, aber auch da fehlten handfeste Belege. Konsulat und Außenministerium spielten die Verantwortungsfrage zurück in die DDR. Zwar sei

25 Ebd., Lux (Ministerium für Bauwesen) an Bambor (AG Entwicklungsländer im Büro des Ministerrats), Berlin, 7.1.1969, Bl. 184.

26 BArch Berlin, DN 1/15578, Nimmrich, Kurzbericht über die Finanzlage in Sansibar, o.O., o.D. [Juli 1967].

27 BArch Berlin, DC 20/11525, Klee, Probleme des Verhältnisses der DDR zu Sansibar, Berlin, 25.10.1967. 
Klee für die Einsatzvorbereitung freigestellt worden, habe dann aber doch den Großteil seiner Zeit mit Vertretungsaufgaben zugebracht, statt sich die dringend notwendigen Englischkenntnisse anzueignen; außerdem, und an dieser Stelle kam die persönliche Ebene ins Spiel, habe es Klee an „Format“ gefehlt. Dem „psychologisch richtigen Umgang mit Afrikanern“ war, wie Klee ebenfalls anmerkte, während seiner Vorbereitung kaum Aufmerksamkeit geschenkt worden. ${ }^{28}$

Was wurde unter dieser Chiffre des „psychologisch richtigen Umgangs“ verstanden? Der erste Berater Gentsch hatte seinen Nachfolgern empfohlen, bescheiden und hilfsbereit aufzutreten und in der gemeinsamen, freundschaftlichen Arbeit jedwede paternalistische Besserwisser- und Hilfshaltung zu vermeiden: „Es sollte keinesfalls zum Ausdruck gebracht werden: Wir helfen Euch. Die Reaktion auf solche Äußerungen ist meist negativ, weil ihnen damit indirekt gesagt wird: Ihr könnt es ja nicht. “29 Gerade in der Anfangsphase sollte der Berater privat wie in der Arbeit „äußerst zurückhaltend sein“, keine „verletzend[en]“ Schlussfolgerungen ziehen und über ,bestimmte Probleme, die hier tiefe Wurzeln haben und über die wir eine gänzlich unterschiedliche Auffassung haben, nicht sprechen, z.B. Religion, Vielweiberei, Rassenprobleme, wie sie sich im Einzelnen abspielen“. ${ }^{30}$ Auch nach der Einarbeitungszeit gelte die Devise, dass ein Berater „keine Weisungen geben kann und darf“, sondern seine Vorschläge am besten so unterbreitet, „dass die Sansibaris vergessen, dass die Vorschläge von ihm kommen und sie als ihre eigenen betrachten“. ${ }^{31}$ Logischerweise stellten etwaige Erfolge dann auch Erfolge der Sansibaris, nicht des Beraters dar. Jegliche Beeinflussung sollte in dieser Hinsicht unsichtbar bleiben. Hinzu kam eine Reihe weiterer Imperative, die den richtigen Umgang mit den Fallstricken der Beratungstätigkeit lehren sollten: Keine Einmischung in Kaderangelegenheiten, keine schematische Übertragung der DDR-Erfahrungen, Ergebnisorientierung statt Papierproduktion, nicht in politische oder Beamtenauseinandersetzungen einbeziehen lassen, die Achtung vor der revolutionären Leistung Sansibars wahren, alle Vorschläge im Hinblick auf ihre Auswirkungen auf die DDR und das sozialistische Lager sowie die Loslösung von der imperialistischen Staaten prüfen und zeigen, „dass wir [...] nicht den Standpunkt der Kolonialherren haben, dass die

28 BArch Berlin, DC 20/11525, Büttner (MfAA) an Dambor (Ministerrat), Berlin, 30.10.1967.

29 BArch Berlin, DN 1/15578, [Gentsch], Ergebnisse auf dem Finanzgebiet in Sansibar, o.O., o.D. [1967], S. 6.

30 Ebd., [Gentsch], Ergebnisse auf dem Finanzgebiet in Sansibar, o.O., o.D. [1967], S. 7, Formatierung im Original.

31 Ebd., S. 8. 
Sansibaris alle träge und dumm sind.“32 Damit ist der typische Anforderungskatalog für Berater in der Entwicklungspolitik aufgestellt: die stets enge und respektvolle Zusammenarbeit mit dem Counterpart, die Überwindung rassistischer Vorurteile, die Selbstzensur bei politisch heiklen Themen (wenn notwendig unter Ausschaltung eigener Ideale), die Wahrung einer Handlungsautonomie gegenüber widerstreitenden lokalen Interessen, das Verbleiben im Hintergrund und die Wahrung der Interessen sowohl des Gast- wie auch Entsendelandes. Fast die gleichen Empfehlungen fanden sich beinah zwei Jahrzehnte später in der Dissertation von Helmut Jung, der in mehreren Ländern als DDR-Regierungsberater gearbeitet hatte. ${ }^{33}$

\section{Die Exekution des Counterparts (1967-1970)}

Ab Mitte 1967 hielt nur noch Finanzexperte Wolfgang Nimmrich eine DDR-Beraterposition in Regierungsnähe aufrecht. Er hatte die - in der Entwicklungsarbeit seltene - Chance, drei volle Monate von seinem Vorgänger Gentsch eingearbeitet zu werden. Zum Zeitpunkt seiner Abreise blickte Gentsch auf eine gut gefüllte Staatskasse und sah Sansibar in das Zeitalter der sozialistischen Moderne eintreten: Von nun an würde die Wirtschaft auf Basis des Fünfjahresplans geleitet. Mit der Abreise der DDR-Planungsgruppe im Frühjahr 1967 wurden Ansätze volkswirtschaftlicher Planung jedoch bereits wieder eingestellt.

Nimmrich, ein Beamter mit über 20 Jahren Erfahrung und seit 1965 Leiter der Organisationseinheit für Wissenschaftlich-Technische Zusammenarbeit im Finanzministerium, hatte die Ausbildung von Sansibaris im Finanzsektor angeleitet und war für die Beziehungen mit „Entwicklungsländern“ zuständig gewesen. ${ }^{34} \mathrm{Er}$ war also im Gegensatz zu Klee hervorragend für den Einsatz geeignet, bekam aber schnell das angespannte Klima zu spüren, das aus der „schlechte[n] Arbeit [der DDR] auf ökonomischem Gebiete“ resultierte und „die Erfolge aus der Beraterund Expertentätigkeit [...] aufzehrte“. Nimmrich vermied jede Erwähnung dieses leidigen Themas gegenüber Twala (,ich hüte mich immer, von mir aus davon

32 BArch Berlin, DN 1/15578, o.A. [Gentsch], Ergebnisse auf dem Finanzgebiet in Sansibar, o.O., o.D. [1967], S. 9.

33 BArch Berlin, DE 1/58117, Helmut Jung, Thesen zur Promotion B: „Zu einigen Erfordernissen und Problemen der Erfahrensvermittlung und der Tätigkeit von Regierungsberatern der DDR in den um ihre nationale und soziale Befreiung kämpfenden Ländern mit sozialistischem Entwicklungsweg“, Maputo, März 1985, S. 4-15.

34 BArch Berlin, DN 1/15578, Mager (Stv. Minister), Beurteilung des Genossen Hans Nimmrich, Berlin, 14.2.1967. 
anzufangen“), der „sehr verärgert“ gewesen sei, dass die DDR sich unter Berufung auf ein $\mathrm{zu}$ hohes wirtschaftliches Risiko vom bereits zugesicherten Bau der Fischverarbeitungsanlage zurückgezogen hatte. Durch diesen Rückzug war Twala selbst in die Schusslinie sansibarischer DDR-Kritiker geraten. ${ }^{35}$ Nimmrich sah bis September 1967, trotz einer zunehmenden „Unausgeglichenheit“ und „Starrköpfigkeit“ Twalas, „keinerlei Hemmnisse“ in seiner Beratungstätigkeit. ${ }^{36}$

Im Oktober 1967 wurde Twala, zusammen mit anderen „progressiven“ ASPMitgliedern, die sich kritisch gegenüber Karume geäußert hatten und z.T. breite Popularität in der Bevölkerung genossen, inhaftiert. Nimmrich berichtete, dass Twala wegen des Verdachts der Veruntreuung staatlicher Gelder vom Revolutionsrat seiner Funktion enthoben wurde, andere Quellen nennen die Bewerbung Twalas für einen US-Studienaufenthalt oder seine Opposition gegen Karumes private Aneignung öffentlicher Mittel (z. B. für die Hochzeit seines Sohnes) als Hintergründe der Festnahme. ${ }^{37}$ Wie auch einige andere Politiker und Funktionäre während der Karume-Ära wurde Twala ohne Prozess exekutiert. Da Nimmrich in einem anderen Schreiben erwähnt hatte, über Twala nur mehr mündlich berichten zu wollen, ist denkbar, dass Nimmrich selbst den sansibarischen (und vom MfS unterstützten) Sicherheitsdienst fürchtete und sensible Informationen fortan zurückhielt - zumindest in der schriftlichen Korrespondenz.

Die DDR-Berater verloren mit Twala ihre wichtigste Informationsquelle und einen unverzichtbaren Transmissionsriemen für Vorschläge an die Regierung. Noch mehr als zuvor waren sie auf das Wohlwollen Karumes angewiesen, dem Nimmrich nun direkt unterstellt wurde. Das kam zwar theoretisch einer Aufwertung gleich, praktisch legte Nimmrich aber ein risikoaverses Verhalten an den Tag und beschränkte sich zunehmend aufs Tagesgeschäft. Sein Vorhaben, weitere Reformen anzustoßen und Bestimmungen aus der Kolonialzeit zu ändern, was er mit Twala hatte angehen wollen, gab er auf, um ,jedes Risiko zu vermeiden“. ${ }^{38}$ Twala war nicht nur kompetent, sondern auch einflussreich gewesen. Nimmrichs neue Hauptpartner, die beide in der DDR ausgebildet worden waren (der Staatssekretär und der Abteilungsleiter Staatshaushalt) und zu denen Nimmrich eine sehr gute Beziehung hatte, waren keine Regierungsmitglieder, wodurch ihr

35 Ebd., Nimmrich an Gentsch, Sansibar, 31.8.1967, S. 3.

36 BArch Berlin, DC 20/11525, Nimmrich, Bericht - Einsatz als Finanzberater in Sansibar vom 5.11.1966 bis 28.2.1969, Berlin, 10.4.1969, Bl. 294.

37 BArch Berlin, DN 1/15578, Nimmrich an Gentsch (MdF), Sansibar, 21.9.1967; Amrit Wilson, The Threat of Liberation. Imperialism and Revolution in Zanzibar, London 2013, S. 80; Burgess, Race, S. 131; Shivji, Pan-Africanism, S. 114.

38 BArch Berlin, DC 20/11525, Hans Nimmrich, Bericht - Einsatz als Finanzberater in Sansibar vom 5.11.1966 bis 28.2.1969, Berlin, 10.4.1969, Bl. 293. 
Wirkungsradius eingeschränkt war. Nimmrich sprach monatlich bei Karume vor, allerdings gegen den „immer stärker werdenden Widerstand des Vorsitzenden des Finanzkomitees, Staatsminister Jumbe“.39 Da der Posten des Finanzministers unbesetzt blieb, konnte Aboud Jumbe seinen Einfluss voll ausspielen und als einer der wenigen unter der schützenden Hand Karumes seine Macht konsolidieren. ${ }^{40}$ Jumbe schob Nimmrich aufs Abstellgleis, sodass dieser klagte, dass Jumbe ,alles $\mathrm{zu}$ wissen glaubt und eine Konsultation mit mir vor einer Entscheidung für unter seiner Würde hält““.11 Im Oktober 1968 beschloss der Revolutionsrat, alle ausländischen Berater sobald wie möglich aus ihren Diensten zu entlassen, was Jumbe den nötigen Rückenwind gab, um für die Verabschiedung des letzten DDR-Regierungsberaters mit dessen Vertragsende am 28.2.1969 zu plädieren. Damit löste sich auch der letzte einflussreiche DDR-Beraterposten (mit Ausnahme der Kooperation im Geheimdienstbereich) in Wohlgefallen auf. Nimmrichs Einschätzung, dass Karume womöglich gern an dem DDR-Berater festgehalten hätte, aber aus innenpolitischen Gründen nicht wagte, „vor dem Revolutionsrat eine Verlängerung des Vertrages zu vertreten“, war plausibel. ${ }^{42}$

Sachliche Gründe für eine Vertragsverlängerung hätte es durchaus gegeben. Sansibars Haushaltslage hatte sich von 1966 bis 1969, vor allem durch den beispiellos hohen Nelkenpreis am Weltmarkt, stetig gebessert. Bis auf Kreditforderungen aus kommunistischen Ländern wie China oder der DDR und kleinere Belastungen aus der vorrevolutionären Ära verzeichnete Sansibar keine nennenswerten Schulden. Trotzdem kam es, da Karume ein Importverbot für Lebensmittel erließ, zu einer Nahrungsknappheit; und die Wirtschaft blieb stark vom Nelkenexport und damit vom Weltmarkt abhängig. Karumes Tirade gegen die ausländischen Finanzberater im Jahr 1970 war somit in mancher Hinsicht eine Projektion von Problemen auf Sündenböcke, die das Land längst verlassen hatten, und die exzellente Finanzlage stärkte Karumes Überzeugung, dass Sansibar imstande sei, seine finanziellen Geschicke ohne externe Unterstützung lenken zu können. TrägerInnen ökonomischen Fachwissens sah er jedoch mit hoher Wahrscheinlichkeit als Gefährdung seines Politikstils. Ein deutlicher Hinweis darauf ist, dass die Wirtschaftsschule, die auf Twalas Initiative gegründet worden war und in der DDR-Lehrer 1966-1968 unterrichtet hatten, von Jumbe ohne Angabe von Gründen am 24.8.1968 geschlossen wurde. Fast alle StudentInnen, die den Zweijahreskurs beinahe abgeschlossen hatten, wurden zu nicht-ökonomi-

39 Ebd., Bl. 295.

40 Wilson, Threat of Liberation, S. 83.

41 BArch Berlin, DN 1/15578, Nimmrich an MdF, Sansibar, 18.12.1967, S. 2.

42 BArch Berlin, Nimmrich, Bericht - Einsatz als Finanzberater in Sansibar vom 5.11.1966 bis 28.2.1969, Berlin, 10.4.1969, Bl. 295. 
schen Auslandsstudien delegiert oder in gänzlich fachfremden Bereichen wie dem Religionsunterricht eingesetzt. ${ }^{43}$ Wirtschaftliche Expertise - und hier ging es wohl eher um eine ökonomische Betrachtungsweise an sich denn um eine spezifisch sozialistische - wurde gezielt marginalisiert, um die politische Entscheidungsmacht nicht einzuschränken.

1972, als das DDR-Engagement bereits weitgehend beendet war, wurde Karume ermordet. Die folgenden Prozesse gegen die Strippenzieher des Attentats waren stark antikommunistisch geprägt, 42 „Genossen“ der früheren UmmaPartei wurden zum Tode verurteilt. ${ }^{44}$ Karumes Nachfolger Aboud Jumbe leitete eine Öffnung Sansibars und Wiederannäherung an das Festland ein; allerdings ohne die Beziehungen mit der DDR wiederzubeleben. Im Gegensatz zu Karumes Beschuldigungen an die Adresse der DDR-Berater sehen historische Studien und zeitgenössische Beobachter - etwa der Politiker Ali Sultan Issa, der als einer der wenigen Verbliebenen der linken Umma-Partei unter Karume einen Kabinettsposten bekleidete - Karumes persönliche Übernahme der Finanzpolitik nach der Exekution von Twala als Wendepunkt zur ökonomischen Misere. ${ }^{45} \mathrm{Zu}$ den Maßnahmen, die zu einer erheblichen Verschlechterung der Versorgungslage führten, zählten die Übernahme des gesamten Einzelhandels durch die staatsnahe Frauenorganisation oder die Auflösung der landwirtschaftlichen Genossenschaften, von denen DDR-Berater explizit abgeraten hatten. Karume und andere Regierungsmitglieder sahen diese jedoch als essenziellen Teil der „ökonomischen Revolution““.46 Der ökonomiezentrierte Gradualismus der DDR-Berater war den soziopolitischen Transformationswünschen Karumes, wohl auch unterstützt von China, nicht gewachsen. Auf weitere tansanische Anfragen für Berater reagierte die DDR jahrelang mit wenig Enthusiasmus.

\subsection{Korruption, Diebstahl und Disziplinierung: Der BRD-Berater im Edelsteinsektor (1970-1974)}

Mitte 1969 erging von Tansania eine Anfrage an die DDR, einen Berater für den Edelsteinsektor zu entsenden. „Trotz ernsthafter Bemühungen“ fand sich kein

43 BArch Berlin, DN 1/15578, Hoffmann, Vorschlag für Errichtung einer Wirtschaftsfachschule in Sansibar, Sansibar, 28.9.1965. Zur Schließung und Versetzungsfragen siehe ebd., Lange, Bericht über den Einsatz in Sansibar, Berlin, 15.10.1968.

44 Wilson, Threat of Liberation, S. 92; Aminzade, Race, S. 203.

45 Burgess, Race, S. 125.

46 BArch Berlin, DN 10/985, Klee an Panse, Sansibar, 1.9.1967. 
geeigneter Kandidat; die DDR musste passen. ${ }^{47}$ Die nächste Anfrage richtete Tansania an die BRD. 1970 trat der westdeutsche Edelsteinberater E. H. Döpel seinen Dienst in Dar es Salaam an und wurde einer der Hauptdarsteller in einem Lehrstück über postkoloniale Politik- und Wirtschaftseliten, das direkt von Issa Shivji zur Illustration seines Konzepts der bürokratischen Bourgeoisie (siehe Kap. 2.4) verfasst worden sein könnte. Döpel erlebte die Selbstbereicherung der Eliten aus nächster Nähe, er sah ihre Verflechtung mit westlichen Firmen und wie diese den wirtschaftspolitischen Zielstellungen Tansanias diametral entgegenstand. Er nahm aber auch die Gegenkräfte wahr, sowohl in der politischen Elite wie auch in den Belegschaften staatlicher Betriebe. Dank seiner Initiative und günstiger politischer Bedingungen konnte sich der westdeutsche Berater einen großen Handlungsradius erarbeiten, schied aber letztlich doch resigniert und vom BMZ der entwicklungspolitischen Wirkungslosigkeit bezichtigt nach vier Jahren aus der Entwicklungsarbeit aus. ${ }^{48}$

\section{Nationalisierung und Korruptionsnetzwerke (1970-1971)}

Als Döpel seine Tätigkeit - er sollte die halbstaatliche National Development Corporation (NDC) in Edelsteinfragen beraten und tansanische Nachwuchskräfte heranbilden - 1970 aufnahm, rollte gerade die zweite Nationalisierungswelle über Tansanias Privatwirtschaft nach 1967 hinweg. Wie die Anfrage an die DDR vermuten lässt, war ein Berater aus einem kapitalistischen in dem finanziell wichtigen und politisch heiklen Edelsteinsektor nicht Tansanias erste Wahl gewesen. Obwohl westliche Unternehmen Anfang der 1970er-Jahre wieder Investitionsvertrauen gewonnen hatten und verstärktes Interesse an den tansanischen Rohstoffvorkommen zeigten, nahm Tansania zeitgleich auch die (letztlich für beide Seiten wenig ertragreichen) Dienste osteuropäischer und chinesischer geologischer Erkundungsdelegationen in Anspruch. Hierbei spielten wohl Motive der pragmatischen Ressourcenakquise genauso eine Rolle wie die aus politischen Gründen angestrebte Diversifizierung der wirtschaftlichen Beziehungen, die

47 PAAA, MfAA, C 773/74, Kattner (MfAA) an DDR-Generalkonsulat in der VRT, Berlin, 19.9.1969. Detaillierte Begründungen für die Ablehnung sind bedauernswerterweise nicht angegeben.

48 Die folgenden Ausführungen basieren, sofern nicht anders angegeben, auf den Projektberichten des Beraters und Korrespondenzen zwischen Botschaft, BMZ und Döpel in BArch Koblenz, B 213/7761 sowie B 213/33046. 
allerdings - wie bekannt - nicht erreicht wurde, sodass es im Bergbausektor bei einer Dominanz westlicher Unternehmen blieb. ${ }^{49}$

Mit der Ankunft Döpels sollten ab 1970 die wichtigsten Edelsteingruben sowie das für die Edelsteinvermarktung zuständige Unternehmen verstaatlicht werden. Die zu diesem Zweck vom Staat gegründete Tanzania Gemstone Industries Ltd. (TGI) hatte die Aufgabe, Einkommen und Devisen zur Finanzierung von Tansanias Entwicklungsplänen zu erwirtschaften - ein staatsmonopolistisches Unternehmen also. Große Hoffnungen setzte die Regierung in einen 1967 entdeckten Edelstein, der nach Bearbeitung als blauer Schmuckstein vermarktet werden konnte und dessen Vorkommen sich auf die Region Arusha beschränkte. Tansania war damit weltweit die einzige Bezugsquelle. Im Einklang mit dem nationalistischen Zeitgeist wurde der Edelstein auf den Namen Tansanit getauft. Da die Diamantvorkommen, deren Abbau bis zu 86\% der jährlichen Edelsteineinnahmen Tansanias ausgemacht hatte, fast erschöpft waren, kam Tansanit umgehend eine Schlüsselrolle für die Zukunft des nationalen Bergbausektors $\mathrm{zu}^{50}{ }^{50}$

Döpel verschrieb sich einer rein wirtschaftlichen Orientierung, ergriff die Initiative und zog schnell immer mehr Aufgaben an sich. Er führte Verhandlungen über die Entschädigung von Privatfirmen für die durchgeführten Nationalisierungen, besuchte potenzielle neue Gruben, trieb die Ausweitung und Rationalisierung der Produktion verschiedenster Edelsteine in Tansania sowie ihre Verarbeitung nach Weltmarktstandards voran und entwarf Pläne, um die Vermarktung zu optimieren. Umtriebig über die nationalen Grenzen hinweg baute er in westlichen und asiatischen Ländern Ruf und Absatz tansanischer Edelsteine aus. Nach einem dreiviertel Jahr bat er die GAWI um Einstufung in eine höhere Gehaltsgruppe, um seiner „faktischen Verantwortung für den ganzen Edelsteinsektor Tansanias“ (,alle Steine [gehen] durch meine Hände“) Rechnung zu tragen. ${ }^{51}$ Schon zu diesem Zeitpunkt hatten sich Spannungen zwischen verschiedenen Parteien aufgebaut.

49 Laut Döpels Berichten stießen sowohl die sowjetische Geologenmission, die mit 80 Mann und schwerem Gerät nach Goldadern suchte, wie auch ein rumänisches joint venture-Vorhaben zur Ausbeutung von Kaolin- und die chinesische Erkundung von Eisenerzvorkommen auf wirtschaftliche oder technische Probleme, zumal auch die sozialistischen Partner nach nüchternen Profitabilitäts- und Rentabilitätskriterien urteilten. Im Falle der UdSSR war Tansania unzufrieden mit den dürftigen Ergebnissen, die zudem nur langsam zugänglich gemacht wurden. Siehe z.B. BArch Koblenz, B 213/33046, Döpel, Tätigkeitsbericht Nr. 5, Dar es Salaam, 4.11.1973.

50 BArch Koblenz, B 213/7761, Zahn (BMZ) an Referat III A 5c, Bonn, 22.8.1972.

51 Ebd., Döpel an BRD-Botschaft, Dar es Salaam, 17.8.1971; ebd., E. Döpel, Bericht Nr. 4, Dar es Salaam, 2.5.1971. 
Ein grundlegender Interessenkonflikt mit der übergeordneten Leitung der National Development Corporation (NDC), deren Tochter die TGI war, entzündete sich an der Frage, mit welcher ausländischen Firma ein Vertrag über die Abnahme des ungeschliffenen, aber profitträchtigen Tansanit geschlossen werden sollte. Der zuvor als Botschafter in Bonn tätige NDC-Generaldirektor George Kahama und der zuständige Abteilungsleiter F. Karim fädelten einen Vertrag mit dem New Yorker Schmuckunternehmen Tiffany ein, deren „skandalös[e] Einkaufspreise“ (Döpel) nur bei einem Drittel des damaligen Weltmarktpreises lagen. Tiffany bot deutlich schlechtere Bedingungen als eine westdeutsche Gruppe - deren Offerte vermutlich auf Döpels Vermittlung zurückging - die deutlich bessere Preise und sogar eine fünfzigprozentige Beteiligung an den Verkaufserlösen geschliffener Steine in Aussicht stellte, ein attraktives und ,bisher einmaliges Angebot in der Geschichte der Edelsteinindustrie“, wie der Berater in seinem Bericht ans BMZ betonte. ${ }^{52}$ Berater Döpel sprach sich daher ausdrücklich gegen den Tiffany-Vertrag aus.

Die Verbindung nach New York, so Döpel, hatte die Firma Litzenberger aus Nairobi hergestellt. Bei der Bonner Vertretung war der Agent Litzenberger eine bekannte Größe für Wirtschaftsbetrug und dubiose Geschäfte zum Nachteil Tansanias, in die auch westdeutsche Unternehmen verstrickt waren. ${ }^{53}$ Generaldirektor Kahama und Abteilungsleiter Karim, der sich bald darauf aus Tansania absetzte, übergingen Döpels Meinung aber genauso, wie sie die staatlichen Gremien umgangen hatten, die Vertragsabschlüsse wie jenen mit Tiffany theoretisch erst abzusegnen hatten. Döpel leitete deshalb eine juristische Prüfung des Vertrages ein. Diesen Vorstoß konnte er wagen, da in Regierungskreisen vermutet wurde, dass der Tansanit-Deal auf geheime Absprachen zwischen den Leitungen der NDC und von Tiffany zurückging und die beteiligten Parteien die Differenz zwischen Einkaufs- und Marktpreis unter sich aufteilten.

\section{Isolierung und politischer Rückenwind (1971-1972)}

Döpels Vorstoß rief wenig überraschend Gegenreaktionen hervor. Aufgrund seiner unliebsamen Stellungnahme setzte die NDC-Leitung einen Briten, einen Vertrauensmann Litzenbergers, als Direktor für den Minenbetrieb ein. Damit war Döpel von der Grubenbegehung und (da die Edelsteine bereits an den Gruben

52 Ebd., B 213/7761, Döpel, Bericht Nr. 3, Dar es Salaam, 14. 2.1971; ebd., Döpel, Bericht Nr. 4, Dar es Salaam, 2.5.1971, S. 2.

53 Ebd., B 213/7675, BRD-Botschaft an AA, Dar es Salaam, 22.9.1971. 
bewertet und sortiert wurden) auch der Preisfestsetzung für das Tansanit „praktisch ausgeschlossen“. Das zwang ihn, seiner wirtschaftlich-fachlichen Orientierung neue Qualitäten beizumengen, um seiner Funktion gerecht werden zu können:

Neben meinem Streben nach größtmöglichem Nutzen für Tansania bin ich bemüht, ein gutes Auskommen mit der Geschäftsleitung zu pflegen, welche mit allen Mitteln versucht, den grösstmöglichen persönlichen Vorteil aus den Edelsteinverkäufen zu ziehen. Diplomatisches Geschick und Geduld müssen hier neben Fachkenntnis gestellt werden. ${ }^{54}$

Diplomatie und Geduld waren freilich schwache Strategien gegen Korruption und Marginalisierung durch zentrale Akteure im Sektor. Die Marginalisierung äußerte sich etwa darin, dass NDC-Direktor Kahama ihm wichtige Unterlagen vorenthielt, wodurch Döpel in seinem Aktionsradius beschnitten wurde und z. B. in Verhandlungen über Investitionskredite für „seine“ TGI erfolglos blieb.

Der politische Wind wehte allerdings für den westdeutschen Berater und bewahrte ihn davor, sich weiter anbiedern zu müssen und tiefer in Kahamas Korruptionsnetzwerke hineinzugeraten. Verstärkt durch die zugespitzte Versorgungslage und Verkündung der neuen Parteirichtlinien (siehe Kapitel 2) regte sich öffentlicher Widerstand gegen staatliche Unternehmen wie die NDC und die Eigenschaften, für die sie mittlerweile standen: Bürokratismus, Ineffizienz, Korruption und Sabotage des tansanischen Sozialismus. Der Tansanit-Vertrag stand symbolisch für all diese Missstände im öffentlichen Sektor. Die Zeitungen, zu diesem Zeitpunkt allesamt regierungs- und parteinah, lancierten Attacken gegen Kahama. Das Ministerium verhängte umgehend nach den ersten Exporten an Tiffany eine Exportsperre für Tansanit und stellte eine absichtlich überzogene Preisrevisionsforderung, wodurch Tiffany sich gezwungen sah, vom Vertrag zurückzutreten. Als politische Reaktion auf die offensichtlich gewordene Macht des NDC-Generaldirektors wurden mehrere Industriezweige aus der NDC ausgegliedert, darunter der Bergbausektor. Dieser Ausgliederung waren andere bereits 1969 vorausgegangen. ${ }^{55}$ Unterstützt durch Finanzminister Amir Jamal bekam die von Döpel vorgeschlagene Vereinigung vier westdeutscher Schleifereien (die im pfälzischen Idar-Oberstein gegründete Trans-Africa Gems) den Zuschlag. Der Vertrag sicherte Tansania nicht nur zu, 10 Prozent des exportfähigen Tansanits selbst vermarkten zu dürfen, sondern garantierte auch um 150 Prozent höhere Preise und damit deutlich höhere Einnahmen. Döpel selbst profitierte ebenfalls

54 Ebd., B 213/7761, Döpel, Bericht Nr. 4, Dar es Salaam, 2.5.1971, S. 2 -3.

55 Coulson, Tanzania, 325-326. 
von den Umstrukturierungen und stieg zum Berater im zuständigen Ministerium für Handel und Industrie auf.

Während wirtschaftliche Erfolge zu verzeichnen waren, gestand Döpel, in den genuin entwicklungspolitischen Aufgabengebieten „Wissenstransfer“ und „Beratung“ der Geschäftsführung weniger Wirksamkeit entfaltet zu haben. ${ }^{56} \mathrm{Genau}$ dies waren aber die feldintern relevanten Bereiche. Das BMZ meldete daher „Zweifel an der Effizienz seines Einsatzes“ an, stimmte dem tansanischen Wunsch um Vertragsverlängerung aber zu: Döpel könne durch seine Versetzung ins Ministerium wichtige Reformen einleiten und „Fehlinvestitionen“ verhindern, zumal die volkswirtschaftliche Bedeutung des Edelsteinsektors noch zunehmen werde. ${ }^{57}$ Dank des politischen Rückenwinds in Tansania konnte Döpel seine Kontakte in die Edelsteinszene - gerade jene in die BRD - voll ausspielen. Über die Vermarktung von Tansanit hinaus plante er in Einklang mit tansanischen Plänen zur vertikalen Integration der Wirtschaft und dem Aufbau von Industrien auf der Basis lokaler Rohstoffvorkommen die Errichtung einer Edelsteinschleiferei in Tansania. Hier griff er wieder auf seine Verbindungen ins pfälzische IdarOberstein zurück, besuchte mit zwei tansanischen Counterparts die dortigen Schleifereien und machte sie mit der Diamantenbörse und KundInnen bekannt. Döpel schickte auch zwei Tansanier zur Ausbildung in die BRD und trieb so den Aufbau eigener Netzwerke mit dem Mehrwert entwicklungspolitischer Nützlichkeit weiter voran. Die Beziehungen zu seinen Counterparts waren jedoch ambivalent.

\section{Disziplinierung und Widerstand (1973-1974)}

Mehrfach lobte Döpel zwar die allgemein gute Zusammenarbeit im Ministerium, beklagte sich aber zunehmend auch über die „unzulängliche Denkweise“, „mechanisches Denken [...], einseitiges Interesse und Lernunwillen“ aufseiten der Counterparts und „Unverständni[s] und Unvermöge[n] des TGI-Management[s]“, weswegen nicht nur eine systematische Counterpartausbildung, sondern auch die Anwesenheit eines Beraters noch auf Jahre erforderlich sei. ${ }^{58}$ Zumindest die Aussagen über die TGI-Geschäftsleitung können keinesfalls als Resultat der (in

56 BArch Koblenz, B 213/33046, Döpel, Tätigkeitsbericht Nr. 1, Dar es Salaam, 29.4.1973; ebd., E. H. Döpel, Tätigkeitsbericht Nr. 3, Dar es Salaam, 30.7.1973.

57 BArch Koblenz, B 213/7761, BRD-Botschaft an AA, Dar es Salaam, 7.6.1972; ebd., Zoller an Referat I B 2, Bonn, 2.8.1972.

58 BArch Koblenz, B 213/33046, Döpel, Tätigkeitsbericht Nr. 1, Dar es Salaam, 29.4.1973; ebd., Döpel, Tätigkeitsbericht Nr. 3, Dar es Salaam, 30.7.1973. 
der Entwicklungsarbeit durchaus häufig anzutreffenden) Desillusionierung oder rein subjektives Urteil angesehen werden. Widerstandspraktiken von Angestellten belegen die Eskalation des Konflikts zwischen Arbeiterschaft und Management. Ende 1973 schied das gesamte technische Personal der TGI aus Protest gegen die Firmenleitung aus; später verwehrte das Personal der Tansanitgruben dem Manager und dem Sicherheitsbeauftragten der TGI gar den Zugang zu den Arbeitsstätten. Diese Praxis des Ausschließens wurde nach Verkündung der neuen Parteirichtlinien von ArbeiterInnen in vielen Betrieben angewendet, um für bessere Arbeitsbedingungen zu kämpfen. Der Sicherheitsbeauftragte wurde wie auch einige andere TGI-Angestellte wegen Korruptionsvorwürfen bald von seinem Posten enthoben und einem Verfahren wegen „Unredlichkeit“ (Döpels Begriff) unterzogen. ${ }^{59}$

Döpel berichtete über Proteste der Angestellten nicht, um Sympathie für die Forderungen der Arbeiterschaft auszudrücken, sondern mit der Intention, die Unfähigkeit der Betriebsleitung zu beweisen. Er setzte sich für die „gerechtere Entgeltung der privaten Edelsteinproduzenten“ (von denen es immer noch einige gab) ein, womit er nicht die breite Belegschaft, sondern die produktionsmittelbesitzende Minderheit meinte. ${ }^{60}$ Wiederholt kritisierte er, dass in Tansania ökonomischen Kriterien weniger Gewicht als politischen Gesichtspunkten beigemessen wurde. Döpel war kein Ujamaa-Anhänger. Er vernahm die „Radikalisierung des politischen Lebens“ und die „gleichmacherische[n] Parolen“, die bis hin zu einer deutlichen Einebnung des Lohngefüges reichten, mit einer distanzierten Nüchternheit; seine eigenen Werte und Haltungen blieben davon unberührt. ${ }^{61}$ Obwohl er die inflationären Tendenzen und die immer schlechter werdende Versorgung mit Grundnahrungsmitteln als materielle Hauptursachen für die allgemeine Unzufriedenheit und die Streiks identifizierte, glichen seine Maßnahmen gegen den verbreiteten Edelsteindiebstahl in den Gruben den Strategien der Regierung und der Gewerkschaftsführung, als sie sahen, dass die wilden Streiks die Produktion ernsthaft gefährdeten. Anstelle einer Anhebung der Löhne veranlasste er die Intensivierung von Kontrollen, statt Selbstbestimmung durch workers committees führte er eine strengere Disziplinierung und die Verschärfung der Sicherheitsbestimmungen ein. Döpel scheiterte jedoch mit seinem Vorhaben, auch auf den höheren Ebenen des Edelsteinunternehmens Kontrollmechanismen wie eine Berichtspflicht einzuführen; selbst die Verluste ausweisende (und damit automatisch für die Leitung unliebsame) Jahresbilanz musste er

59 Ebd., S. 3.

60 BArch Koblenz, B 213/7761, Döpel, Bericht Nr. 10 (Schlussbericht), Frankfurt/Main, 16.9.1972.

61 Ebd., Döpel, Bericht Nr. 5, Dar es Salaam, 2.8.1971. 
1973 ohne jede Zuarbeit allein erstellen. Die persönlichen Lagerstätten- und Grubenbegehungen gehörten zu seinen regelmäßigen Beschäftigungen, da er annahm, dass nur die Anwesenheit ausländischer Experten den Produktionsrückgängen in den Edelsteingruben entgegenwirken könnte. ${ }^{62}$

In Berücksichtigung der Debatten der frühen 1970er-Jahre ist denkbar, dass die Arbeitskräfte in den Minen Döpels Inspektionsbesuche einerseits als Fortsetzung kolonialer Arbeitsverhältnisse deuteten (und kritisierten), zumal seine Disziplinierungsversuche auch auf die Einschränkung ihrer Autonomie und Bereicherungsmöglichkeiten abzielten. Andererseits mochten sie in ihm auch ein Gegenbild zu den korrupten Eliten wie Kahama und lokalen Vorgesetzten sehen, einen expatriate, der ihre Macht einschränken oder sie gar zur Rechenschaft ziehen konnte. Döpel jedenfalls sah in Tansania einen Mangel an Vorarbeitern, die die Arbeiterschaft seinen Vorstellungen entsprechend disziplinieren konnten. Er veranlasste daher das Bergbauunternehmen, zwei BRD-Experten mit Erfahrung in der Untertageproduktion aus der BRD anzufordern, was vom BMZ offensichtlich gar nicht erst diskutiert wurde und auch unter entwicklungspolitischen Gesichtspunkten kaum argumentierbar gewesen wäre.

Döpels ökonomistische Orientierung hatte nicht verhindern können, dass Ende 1973 die „Geschäftslage und [die] personelle, insbesondere technische Besetzung der TGI ... einen Tiefstand“ erreichten. ${ }^{63}$ Döpels Rationalität - oder womöglich doch seine Loyalität zu seinen bundesdeutschen Handelspartnern sollte noch ein letztes Mal zum Vorschein kommen, als deutsche und tansanische Parteien 1974 den Tansanitpreis neu verhandelten. Er hatte die Verhandlungen mit vorbereitet, aber als diese scheiterten, meinte er, das liege an Tansanias „unrealistische[n]“ Preisvorstellungen, für deren Durchsetzung er sich offensichtlich nicht zuständig sah. ${ }^{64} \mathrm{Zu}$ diesem Zeitpunkt hatte Döpel bereits seit einigen Monaten das Interesse an seiner Tätigkeit verloren, ebenso wie seine Counterparts an ihm. Keine Seite setzte sich für eine Vertragsverlängerung ein. Tansania fragte jedoch um einen Nachfolger für Döpel an, das zuständige Ministerium muss also einen Nutzen im Beraterposten gesehen haben. Wahrscheinlich ging es um die Handelskontakte, deren Ausweitung mit dem Verhandlungsstop an ihre vorläufige Grenze gelangt war. Die BRD antwortete zurückhaltend auf die Anfrage: Sobald Tansania einen Experten für eine Anstellung auf Basis eines lokalen Vertrags rekrutiert hätte, sollte ein Antrag auf Gehaltsaufstockung gestellt werden. Damit endet die Archivspur; das „Projekt“

62 BArch Koblenz, B 213/33046, Döpel, Tätigkeitsbericht Nr. 3, Dar es Salaam, 30.7.1973, S. 3.

63 Ebd., Döpel, Tätigkeitsbericht Nr. 5, Dar es Salaam, 4.11.1973, S. 2.

64 Ebd., Döpel, Tätigkeitsbericht Nr. 6, Dar es Salaam, 4. 8.1974, S. 2. 
Edelsteinberatung blieb mit vier Jahren Dauer eins der kürzeren der bundesdeutschen Technischen Hilfe.

An der relativ gut, aber fast nur einseitig dokumentierten Tätigkeit Döpels zeigt sich, wie wichtig politische Konjunkturen für die Beratertätigkeit sind. Ohne die politische Großwetterlage und konkrete ministerielle Unterstützung, dank der die Korruption in der NDC bekämpft und das Edelsteinunternehmen aus der NDC ausgegliedert wurde, wäre Döpel in seinem Spielraum äußerst eingeschränkt geblieben. Döpel selbst zeichnet das Bild eines erst erfolgreichen, später aber gegen Windmühlen kämpfenden Einzelkämpfers. Gegen das Einzelkämpfernarrativ spricht, dass erfahrenes tansanisches Personal (übernommen vom vorherigen privaten Unternehmen) und andere westliche und osteuropäische Experten in seinem Umfeld tätig waren, deren Einfluss in den Berichten aber schemenhaft bleibt. Während Döpel die personellen Verfilzungen zwischen NDC, Tiffany und Litzenberger aufdeckt, bleiben seine eigenen persönlichen Netzwerke im Dunkeln. Damit wahrte er das Bild des technokratischen, nur an der Sache interessierten Beraters. Dabei könnte Döpels für einen Berater äußerst unkonventionelle Konfrontationsstrategie mit einer juristischen Untersuchung gegen die Korruption anzugehen, mit eigenen Profitbeteiligungen bei den BRD-Geschäften zusammengehangen haben. Sein proklamiertes Hauptziel waren schwarze Zahlen für das Unternehmen, nicht der Aufbau einer Edelsteinindustrie, die mit UjamaaPrinzipien oder gar den Forderungen des Mwongozo kompatibel war. Da aber auch in der tansanischen Elite die devisenbringende Funktion des Edelsteinsektors im Vordergrund stand, war das kein Reibungs-, sondern ein gemeinsamer Ausgangspunkt. Die Arbeitskräfte wiederum konnten sich den Disziplinierungen offensichtlich oft entziehen, wie die sinkende Produktivität und Rentabilität fast aller Gruben zeigte. Das staatliche Handelsmonopol wurde zudem, wie auch im Fall von Diamanten, durch Schmuggel umgangen.

Ob Döpels Verbindungen in das international bedeutende Edelsteinzentrum Idar-Oberstein für Tansania ein tatsächlich so spektakulär gutes Angebot bedeuteten, wie er suggerierte, muss hier offenbleiben. Ein hypothetischer, kontrafaktischer Vergleich mit dem angefragten DDR-Berater ist aber nützlich: Was wäre im Fall eines ostdeutschen Edelsteinspezialisten anders gewesen? Ein DDREntsandter wäre kaum auf die Idee gekommen, eine juristische Untersuchung gegen faktische Vorgesetzte einzuleiten. Obwohl es auch in der DDR in Edelsteinfragen qualifizierte Fachleute gab und vielleicht sogar einige mit Außenhandelserfahrungen und einem profitorientierten Habitus, ist es doch schwer vorstellbar, dass ein ostdeutscher Berater erfolgreich durch das westliche und asiatische „nicht-sozialistische Wirtschaftsgebiet“ gezogen wäre, um wie Döpel neue Handelspartnerschaften aufzubauen - zumal ihm (oder ihr) die GAWI-Devisen gefehlt hätten, mit denen Döpel seine Reisen zum Aufbau sozialen Kapitals 
erst realisieren konnte. Ebenso hätte wohl kein DDR-Unternehmen Tansanit gegen Devisen abnehmen können, womit die Anbahnung von Handelsbeziehungen von vorneherein ausgeschlossen gewesen wäre. Es ist schwer zu sagen, was Tansania mit der Anforderung eines DDR-Edelsteinfachmanns bezweckt hatte - womöglich aber tatsächlich nur die Ausübung der Beratungstätigkeit im nationalen Rahmen, über die Döpel dann weit hinausging.

\subsection{Neokoloniales Management: BRD-„Berater“ bei der National Engineering Company (ca. 1979-1981)}

Zeitgleich und im Anschluss an Döpel wurden weitere westdeutsche Experten in „Beratungsprojekten“ im Finanz- und Wirtschaftssektor im Rahmen der Technischen Hilfe eingesetzt. Sie sollten helfen, den staatlichen Wirtschaftssektor mit aufzubauen beziehungsweise, als sich dieser als zunehmend marode erwies, zu sanieren. Rekrutiert wurden die ersten Berater von der Consultingfirma Africa Bureau Cologne - Gesellschaft für Entwicklungsplanung. Einige von ihnen kamen mit Vorerfahrungen aus der Deutschen Entwicklungsgesellschaft, deren Angestellte bei Projektentscheidungen in erster Linie darauf schauten, ob sich die Vorhaben „rechneten“.65 Die Experten selber setzten auf Profitabilität und Produktivität. Betriebs- und volkswirtschaftliche Zahlen waren für sie die wichtigsten Erfolgsindikatoren; soziale Kriterien spielten eine bestenfalls untergeordnete Rolle. Dieser Habitus war dem wirtschaftlichen Feld zugehörig und vom zeitgenössischen entwicklungspolitischen Trend zur Grundbedürfnisorientierung weit entfernt. Die Anlaufschwierigkeiten und Missstimmungen, die „Reserviertheit und teilweise sogar Obstruktion“, auf die viele dieser Berater anfangs in Tansania stießen, schob die GTZ mit dem Verweis beiseite, dass ein „Sanierungsfachmann“ oder gar „Konkursverwalter“ ja ,auch in Europa [...] keineswegs mit offenen Armen empfangen“" werde. ${ }^{66}$

Die Projekte setzten zwischen 1972 und 1977 ein, wobei zwischen 1975 und 1978 zunehmend ganze Teams statt einzelner Berater entsandt wurden. In der National Transport Company und in der National Engineering Company etwa waren teils mehr als zehn Experten tätig. Die politisch gewandelten Prioritäten und Kräfteverhältnisse Mitte der 1970er-Jahre zeigten sich darin, dass die „Berater“ alle auch Exekutivfunktionen in sensiblen Bereichen an der Schnittstelle von

65 Manfred Glagow u. a., Die deutschen Entwicklungsbanken. Zur Organisation und Tätigkeit der Kreditanstalt für Wiederaufbau (KfW) und der Deutschen Finanzierungsgesellschaft für Beteiligungen in Entwicklungsländern (DEG), Saarbücken 1985, S. 60, S. 242-244.

66 BArch Koblenz, B 213/33065, GTZ an BMZ, Eschborn, 10.5.1978, S. 2. 
Finanz-, Privat- und staatlichem Wirtschaftssektor wahrnahmen. Expatriates kehrten in diesen Jahren zunehmend in Entscheidungsfunktionen zurück. 1974 waren nur 8 von 24 Managementposten in NDC-Firmen mit einheimischen Fachkräften besetzt; in 14 von 16 Fällen waren die Besetzungen der Positionen mit expatriates an internationale Abkommen gebunden, in denen externe Interessen eine große Rolle spielten. Die Regierungsmaßnahme Operation Leapfrog, die zur personellen Re-Nationalisierung der Unternehmen führen sollte, schlug fehl. Die Vorgabe, tansanische Counterparts auszubilden, damit diese die expatriates in den Unternehmen ersetzten, zeitigte kaum Auswirkungen. ${ }^{67}$

GTZ-Experten evaluierten Projekte für die Tanzania Investment Bank (ein Finanzierungsinstitut, das u.a. externe Kapitalhilfe durchleitete), kontrollierten Investitionen und berieten private Unternehmen für die Tanganyika Development Finance Company (eine international finanzierte Entwicklungsgesellschaft zur Förderung privater Investitionen). Eine weitere Gruppe übernahm die finanzielle Evaluierung und Projektdurchführung für die bereits erwähnte National Development Corporation, eine Staatsholding im Industriebereich, die sich aus Regierungsgeldern und ausländischen Mitteln finanzierte. ${ }^{68}$

Die lakonischen Berichte der „Berater“ bieten wenig Greifbares: Akteure und Konflikte wurden kaum zur Sprache gebracht. Nur auf ihrem ureigenen Feld, dem Rechnen, gaben sie konkrete Urteile ab. Diese vielen auffällig häufig so aus, dass externe Angebote - gleich ob von Firmen aus kapitalistischen Ländern oder Staatsunternehmen aus Osteuropa - in der Regel überdimensioniert, überteuert und den eigenen Export- und Profitinteressen, nicht aber den tansanischen Bedingungen und Bedürfnissen angepasst waren. Ihre eigene Haupttätigkeit sahen die Experten dann darin, Tansania vor aufgeblasenen Projekten und daraus erwachsenden, unnötig hohen Rückzahlungsverpflichtungen zu bewahren. Mehrmals rechtfertigten sie ihre Tätigkeit dadurch, dass Tansania aufgrund ihrer Angebotsanalysen Millionen von tansanischen Schilling „gespart“ habe. ${ }^{69}$ Wie Edelsteinberater Döpel bekamen aber auch sie vom BMZ oft vorgeworfen, sie

67 A. S. Nsekela, The Public Enterprise an Instrument of Economic Development in Tanzania, in: Gabriel Ruhumbika, Hg., Towards Ujamaa: Twenty Years of TANU Leadership. A Contribution of the University of Dar-es-Salaam to the 20th Anniversary of TANU, Nairobi, Kampala, Dar es Salaam 1974, S. 108-147, hier: S. 145. Für die Perspektive eines US-Amerikaners, der damals eine Studie in Tansania zur Operation Leapfrog durchführte, siehe David A. Emery, From Kansas to Kilimanjaro: A Memoir of a Family That Survived Two World Wars and Outwitted Russian Espionage, Bloomington 2012, S. 92-96.

68 BArch Koblenz, B 213/33056, Africa Bureau Cologne an BfE, Köln, 29.10.1974, S. 2 -3.

69 BArch Koblenz, B 213/33088, Scholwin, Projektfortschrittsbericht 1/80, Dar es Salaam, 5.5. 1980. 
seien an der essenziellen Aufgabe der Counterpartausbildung gescheitert und hätten eine $\mathrm{zu}$ geringe entwicklungspolitische Wirkung gezeigt.

Statt einer ganzen Projekt- oder Einsatzchronologie steht im Folgenden ein Konflikt im Vordergrund, der sich 1980/81 um die Fortführung des Beratungsprojektes bei der National Engineering Company (NECO) abspielte. Durch die Auseinandersetzungen ist die Quellenlage in diesem Zeitraum durch eine besondere Dichte und Vielstimmigkeit gekennzeichnet. Die Analyse offenbart einerseits die Bildung einer Allianz im „Entwicklungsgeschäft“, die sich sogar gegen das BMZ durchsetzen konnte und andererseits, dass tansanische Counterparts die „Berater“ nicht in erster Linie für ihre fachlichen Kenntnisse brauchten, sondern für die Kontakte, Ressourcenflüsse und das symbolische Kapital, das mit den Personaleinsätzen verknüpft war.

\section{„Eine vollendete Katastrophe“: Das Projekt 1980 am Scheideweg}

1980, als das Beratungsprojekt bei der National Engineering Company in sein fünftes Jahr ging, schlug die Botschaft Alarm: Es sei „eine vollendete Katastrophe“ und würde bei einem sofortigen Abbruch nichts als „eine rauchende Ruine“ hinterlassen. Als Hauptproblem galt „eine absolute Dominanz der deutschen Seite“ durch die massive Präsenz des GTZ-Personals, die in dieser vergleichsweise kleinen halbstaatlichen Institution mit etwa 300 Beschäftigten einen Grad erreicht hatte, durch den „Tansanier geradezu herausgedrängt“ und sich auf ausgeschriebene Counterpart-Stellen gar nicht erst bewerben würden. ${ }^{70}$ In Hinblick auf Tansanias akute Devisennot, die zu Importstopps und Produktionsausfällen führte, sollten die Gelder in Waren- statt Personalhilfe gesteckt werden. Der deutsche Personalstamm hatte 1975 aus einem einzigen integrierten Experten bestanden und war sukzessive auf elf Fachkräfte angewachsen, die allesamt nur dem Namen nach Berater waren. Der gleichzeitig als General Manager fungierende Projektleiter Deil vereinte ,absolute Weisungsbefugnis über NECO und das Expertenteam“ und trug die Verantwortung für den kaufmännischen Bereich und das Personalwesen. ${ }^{71}$ Das BMZ schrieb von Beratern nur in Anführungsstrichen und führte umständliche Formulierungen wie „Berater in Linienfunktion“ und

70 BArch Koblenz, B 213/33089, BRD-Botschaft an AA, Dar es Salaam, 18.7.1980; ebd., B 213/ 33088, Küper (GTZ), Besprechungsbericht: Entwicklungspolitische Zusammenarbeit mit Tansania, Eschborn, 14.11.1980.

71 BArch Koblenz, B 213/33089, P. E., Projektbericht (Zeitraum 15.9.1978 - 31.7.1980), o.O., 29.8. 1980, S. 15. 
„Berater [...] als verantwortlich Entscheidende“ ein, mit denen die Abkehr vom Beratungsideal nur notdürftig verschleiert wurde. ${ }^{72}$

Ganz im Gegensatz zum Nationalisierungsimperativ der frühen 1970er Jahre sah das tansanische Management der NDC die westdeutsche Präsenz nicht als Problem. Es war das BMZ, das mit entwicklungspolitischen Argumenten auf die „Tansanisierung“ drängte. Als BMZ-Beamte 1981 den Druck erhöhten, den Personaleinsatz schleunigst $\mathrm{zu}$ beenden und durch einen reinen Finanzierungsvertrag zu ersetzen, gingen nicht nur der GTZ-Projektleiter und das GTZ-Hauptquartier, sondern auch die NDC-Leitung, tansanische Ministerien, Gewerkschaftsund Parteivertretungen im Betrieb und selbst die zuvor kritische BRD-Botschaft auf die Barrikaden. Diese breite Allianz übte massiven Widerstand gegen die Umstrukturierungspläne des BMZ aus und forderte den Fortbestand des ,deutsche[n] Management[s]“". ${ }^{73}$ Hinter dieser Forderung standen akteursspezifische Motive, deren wichtigster Überschneidungspunkt ökonomische Argumente waren.

NDC-Vorsitzender und Geschäftsführer Arnold Kilewo war oft in der Welt unterwegs, um Investitionsgelder für Entwicklungsvorhaben und Personal für eine effiziente Produktion einzuwerben. Schon der Betrag von 8,9 Mio. DM, den die BRD allein für die erste Phase des „Beratungsprojektes“ zugesagt hatte, überstieg das NECO-Eigenkapital um mehr als Achtfache, wenngleich der Großteil des Budgets für die Bezahlung des deutschen Personals verwendet wurde. Die GTZ-Personalentsendung ging mit einer umfassenden „Warenhilfe“ als Investition in gesteigerte Produktionskapazitäten (u.a. eine neue Gießerei) einher. Da die NECO als Tansanias einziges Stahlbauunternehmen „eine monopolartige Schlüsselfunktion“ einnahm, hatte sie eine große wirtschaftliche Bedeutung auf nationaler Ebene. ${ }^{74}$ Mit den extremen Devisenengpässen ab 1979 nahm diese Bedeutung in finanzieller und exportstrategischer Hinsicht weiter zu: Alle von NECO produzierten Güter bedeuteten eingesparte Devisen; durch den Aufbau eigener Kapazitäten sollten Importe auch in langfristiger Perspektive substituiert und so die Zahlungsbilanz entlastet werden. ${ }^{75}$ Die Counterparts auf „Empfängerseite“ belegten ihr genuines Interesse dadurch, dass sie in den tansanischen Haushaltsverhandlungen ein Investitionsbudget erkämpften, das in diesen Jahren der Wirtschaftskrise nur wenigen Institutionen vorbehalten war. Wiederum war

72 Ebd., Kremer (BRD-Botschaft) an AA, Dar es Salaam, 19.6.1981; ebd., Wüstermann (BMZ) an Referat 114, Bonn, 4.2.1976.

73 BArch Koblenz, B 213/33100, Wiegmann (BMZ) an Referat 122, Bonn, 11.9.1981.

74 Ebd.

75 Darunter eine Reihe von Erzeugnissen, die im Rohstoffabbau und anderen Industrien benötigt wurden, z. B. Walzenballen, Hohlträger, Schachtdeckel und Räder. 
der politische Rückenwind dafür entscheidend: Nach dem Scheitern der landwirtschaftlichen Transformationsstrategie (1969-75) lag ein politischer Fokus nun auf dem Aufbau einer eigenen Grundstoffindustrie. Diese Strategie war ein Kernstück des dritten Fünfjahresplans (1976-81) und stark vom dependenztheoretischen Denken des Guyaners Clive Y. Thomas und dem tansanischen Wirtschaftswissenschaftler Justinian Rweyemamu beeinflusst, die beide auch an der Universität Dar es Salaam unterrichteten. ${ }^{76}$

Wären jedoch nur Warenhilfe und Investitionen für die NDC-Führung entscheidend gewesen, hätte sie keinen Grund gehabt, weiter auf die Präsenz westdeutschen Personals zu setzen. Dessen Wichtigkeit ergab sich aus der Verknüpfung mit privaten Wirtschaftsinteressen. Ab 1978 versuchte die GTZ verstärkt, Geschäftsbeziehungen zwischen NECO und westdeutschen Firmen herzustellen. Diese sollten den Know-how-Transfer erleichtern (wobei das eigentliche Hauptproblem, nämlich das Fehlen von Counterparts, dadurch nicht im Geringsten berührt wurde). NDC-Geschäftsführer Kilewo flog 1980 mit dem NECO-Geschäftsführer und GTZ-Projektleiter Deil in die BRD, um Kontakte zu den Unternehmen Noell, Mannesmann-Demag, Henschel-Thyssen und Krupp herzustellen. Diese Firmen wollten auf dem tansanischen Markt Fuß fassen, zogen eine Kooperation allerdings nur in Betracht, solange (jedenfalls vorläufig) ein ,deutsches Management“ vorhanden war. ${ }^{77}$ Auch der „externe“ GTZ-Gutachter (gleichzeitig der Bruder eines im Projekt angestellten Experten) sah die deutsche Wirtschaftsexpansion und Entwicklungshilfe wie einst BMZ-Minister Walter Scheel in den 1960er-Jahren als zwei Seiten derselben Medaille:

Es ist zwar nicht Aufgabe der Entwicklungshilfe [...], der deutschen Industrie zu neuen oder größeren Gewinnen zu verhelfen, andererseits ist aber eine deutsche Niederlassung oder Beteiligung im Entwicklungsland eine logische Fortsetzung der vom BMZ begonnenen Maßnahmen. ${ }^{78}$

In Tansania selbst hatten die Auftraggeber der NECO, bei denen es sich oft um expatriates aus Indien und westlichen Ländern handelte, dem Bericht des Gutachters zufolge kaum mehr Vertrauen in ein tansanisches Management. Etwa die Hälfte aller Aufträge der NECO wären „davon abhängig gemacht“ worden, „daß

76 Coulson, Tanzania, S. 360 - 361; Clive Y. Thomas, Dependence and Transformation: Economics of the Transition to Socialism, New York 1974; Justinian Rweyemamu, Underdevelopment and Industrialization in Tanzania, Nairobi 1973.

77 BArch Koblenz, B 213/33088, Lütteken (BMZ), Vermerk, Bonn, 30.7.1980, S. 2.

78 BArch Koblenz, B 213/33089, Elmering, Projektbericht (Zeitraum 15.9.1978 - 31.7.1980), o.O., 29.8.1980, S. 31. 
das Management mindestens vorläufig von deutschen Experten gestellt wird“. ${ }^{79}$ Die NECO war also im Laufe des „Beratungszeitraums“ ökonomisch vollständig von der deutschen Präsenz und dem „Standing“ als GTZ-Projekt abhängig geworden. Die Warenhilfe sowie die Patente und Halbfertigprodukte, die NECO verwendete, kamen ohnehin größtenteils aus der BRD. ${ }^{80}$ Der Gutachter empfahl, das Projekt schon deswegen weiterzuführen, weil Tansania mit eigenem Geld in deutsche Maschinen investiert hatte und bei einem Rückzug der rufschädigende Vorwurf kapitalistischer „Geschäftemacherei“ unvermeidlich sei. ${ }^{81}$

Die Präferenz für ein „deutsches Management“ wird aber erst voll verständlich, wenn auch nicht-ökonomische Faktoren miteinbezogen werden. Staatssekretär Mujuni aus dem tansanischen Industrieministerium meinte - sich auf seine Erfahrung berufend - dass die ,vorzeitige [...] Übertragung der Verantwortung auf die Tansanier regelmäßig zum Zusammenbruch“ führe, was auch im Falle von NECO zu erwarten sei. Wahrscheinlich teilte er auch die Furcht der Deutschen (die kritischen BMZ-Beamten freilich ausgenommen), dass bei einem Managementwechsel die Partei- und Gewerkschaftspolitik die Betriebsführung bestimmen und die NECO „Zugrunderichten“ würden. ${ }^{82}$ Befürchtet wurde hier also nicht primär der Expertiseverlust. Es ging um politische Entscheidungsmacht. Das wird an zwei Punkten deutlich. Erstens ist die Argumentation nicht ganz widerspruchsfrei, denn einerseits wurde berichtet, dass Partei und Gewerkschaft ebenfalls stark für ein fortgesetztes deutsches Management plädierten; andererseits gab die GTZ zu bedenken, dass die NDC als Mutterorganisation der NECO wohl auf „kurzfristige Gewinnmaximierung“ statt auf die Ausbildungsfunktion setzen würde. ${ }^{83}$ Gerade den letzten Vorwurf konnte man deutschen Experten aber ebenso machen. Außerdem wäre selbst bei einem Finanzierungsbeitrag weiterhin ein deutsches Consultingteam vor Ort geblieben; nur das Management wäre in tansanische Verantwortung übergegangen. Die Consultinglösung war für die westdeutschen Experten jedoch inakzeptabel.

79 BArch Koblenz, B 213/33100, Tillmanns, Bericht über den Projektbesuch vom 4. bis 18. 8.1981, Niddatal, 26.8.1981, S. 7.

80 DAAD-Archiv, B 212/91395/1, KAS (Bereich IIS-Auslandsinformation), Erfolge und Grenzen deutscher Entwicklungspolitik (Teil 2), 15.6.1984, S. 12-13.

81 BArch Koblenz, B 213/33089, Tillmann, Stellungnahme zum Stand des Projektes für die GTZ, o.O., Januar 1981, S. $9-10$.

82 BArch Koblenz, B 213/33100, BRD-Botschaft an AA, Dar es Salaam, 4.9.1981.

83 BArch Koblenz, B 213/33089, Hanusch (GTZ), Projektsprecherbericht, Eschborn, 27.4.1981, S. 6. 


\section{Pseudo-nationale Interessen und das Prestige der Technischen Hilfe}

An dieser Stelle kommt der Habitus der GTZ-Experten ins Spiel. Diese sträubten sich, ein direktes Vertragsverhältnis mit der NDC einzugehen und wollten „um jeden Preis einen deutschen Arbeitgeber behalten““ ${ }^{84}$ Hier ging es um Prestige, Einfluss und wirtschaftliche Interessen, da die eigenen Karriere- und Verdienstvorstellungen von den Diskussionen um den Projektstatus (Technische Hilfe oder Finanzhilfe, Führung des Unternehmens oder Consulting) in Mitleidenschaft gezogen werden konnten. Bei den „Beratern“ handelte es sich nicht um fest angestellte GTZ-Kräfte, die meisten hatten auch keine vorherige Erfahrung in der Entwicklungsarbeit vorzuweisen. In einem beispiellos komplizierten Arrangement hatte die GTZ den Auftrag zur Projektdurchführung an eine Arbeitsgemeinschaft vergeben, die sich aus drei verschiedenen Ingenieurbüros zusammensetzte. Die Experten standen also einerseits im Dienst der GTZ mit einer entwicklungspolitischen Rationalität, gleichzeitig aber auch für ihre jeweiligen Consultingfirmen, für die sie weitere Aufträge akquirieren sollten. ${ }^{85}$ Aus persönlicher Perspektive war ein Anstellungsverhältnis bei der NECO unsicherer und in finanzieller Hinsicht deutlich weniger lukrativ als ein Vertrag im Rahmen der Technischen Hilfe: anstelle der kompletten Befreiung des Gehaltes von Steuern drohte die Doppelbesteuerung in Tansania und der BRD, weitere Zusatzleistungen würden ebenfalls entfallen.

Darüber hinaus war auch „Gefühlsmäßiges im Spiel“, wie die GTZ-Zentrale einräumte: Die Experten hatten angekündigt, sie „wären mit einer Verschlechterung ihres persönlichen Status nicht einverstanden“, da sie sich als Vertreter der BRD und nicht als NDC-Angestellte sahen. ${ }^{86}$ Das GTZ-Hauptquartier und die Entsandten fürchteten eine „Schwächung der [deutschen] Position“; wohingegen die BMZ-Beamten nationale Interessen als illegitimes Argument ansahen und klarstellten: „[I]n einem tansanischen Betrieb haben wir keine deutsche Position zu verteidigen“. ${ }^{87}$ Davon abgesehen seien Experten - ein deutlicher Gegensatz zu DDR-Auslandskadern - keine Vertreter der BRD. Die „deutsche Position“ war also eine Redensart, die sich die Experten und die GTZ aus Argumentationsgründen aneigneten. Statt um „nationale Interessen“ der BRD ging es jedoch um ihre Partikularinteressen. Projektleiter Deil instrumentalisierte seinen Status als BRDVertreter (zwar nahm ihn das BMZ nicht als solchen wahr, andere Akteure aber durchaus) zum Ausbau seiner bereits weitverzweigten Netzwerke. Oft und gern

84 BArch Koblenz, B 213/33100, Wiegmann (BMZ) an Referat 122, Bonn, 11.9.1981, S. 2.

85 BArch Koblenz, B 213/33089, Wiegmann (BMZ) an GTZ, Bonn, 21.1.1981, S. 5.

86 Ebd., Hanusch (GTZ), Projektsprecherbericht, Eschborn, 27.4.1981, S. 7-8.

87 Ebd. 
verwies er auf seinen Einfluss in deutschen Großfirmen, in evangelischen Kirchenkreisen, in der UNCTAD und seinen Kontakt zu einem Bundesverfassungsrichter (den er sogar nach Dar es Salaam gelotst hatte). Ihm zufolge war das „Prestige“ eines Projekts der Technischen Hilfe „wichtig, dass wir uns hier als Projekt der GTZ, d.h. letztlich der Bundesregierung gerieren können“; er könne gegenüber Institutionen wie der Weltbank unmöglich „als Angestellter irgendeiner Consulting oder gar der NDC auftreten““ ${ }^{88}$ Deil versuchte auch den Minister für wirtschaftliche Zusammenarbeit Offergeld persönlich durch einen Brief und Gespräche bei dessen Besuch in Dar es Salaam zu einem Machtwort gegenüber den störrischen BMZ-Beamten zu bewegen, blieb damit aber vorerst erfolglos.

Angesichts der materiellen Interessen und der zwischengeschalteten Ingenieurbüros gilt hier ganz besonders, dass die Berichte (die stets um Monate zu spät abgeliefert wurden) stark gefiltert waren. Erst Konflikte innerhalb des Beraterteams führten dazu, dass auch für die Entscheidungszentralen in Bonn und Eschborn weitere Standpunkte sichtbar wurden. Schiele (Name geändert, E. B.), einer der Experten, deren Vertrag in Dar es Salaam nicht verlängert wurde, nahm den Weg nach Bonn zum BMZ auf sich, um dort persönlich - aufgrund manipulierter Berichte „erfahre [die GTZ] ja auch nicht, was los sei“ - eine Kaskade von Vorwürfen auf den Projektleiter und seine engsten Vertrauten niedergehen zu lassen. Die Vorwürfe stellten sich im Verlaufe weiterer Nachforschungen als begründet heraus. Fachlich sei der Einsatz „für die Katz“ gewesen, u. a. weil Projektleiter Deil Schieles Counterpart abgezogen und auf eine andere Position versetzt habe, wodurch Schieles Abteilung nach dessen Einsatzende komplett zusammengebrochen sei. Schiele warf Deil darüber hinaus vor, gegen Dienstvorschriften verstoßen $\mathrm{zu}$ haben. Während einer zweimonatigen Abwesenheit hatte Deil seiner Frau den Dienstwagen zur Verfügung gestellt; außerdem habe Deil Schiele dazu abkommandiert, Reparaturen an diesem Wagen vorzunehmen, was nicht seinem Einsatzzweck entspreche. Ein anderer Experte im Projekt sei wegen der schlechten Auftragslage zeitlich nie ausgelastet gewesen und regelmäßig „mit dem [Projekt-]Auto voller Fleisch“ von der Jagd zurückgekommen. Schiele deckte auch Klüngeleien auf: Der GTZ-Gutachter war der Bruder eines Experten, beide hatten ein enges Verhältnis zu Deil. Die Ingenieurbüros hatten alle bis auf einen Mitarbeiter eigens für den Tansania-Einsatz angestellt, ,also nur Personal-Leasing“ betrieben. Einer der Rekrutierten zahlte laut Schiele monatlich 1.000 DM an einen Kollegen für die Vermittlung (dies war der einzige Vorwurf, den Schiele später zurückzog, allerdings nur, weil ihm belastbare Beweise fehlten). Das BMZ forderte Aufklärung von der GTZ, die ihrerseits alle Kritikpunkte her-

88 BArch Koblenz, B 213/33089, Wiegmann (BMZ) an Minister Offergeld, Bonn, 30.6.1981, S. 3. 
unterspielte. ${ }^{89} \mathrm{Zu}$ Konsequenzen oder gar Disziplinierungsmaßnahmen scheint es nicht gekommen zu sein.

Die Botschaft hatte in ihrem alarmierenden Bericht 1980 argumentiert, dass es vor allem die massive Präsenz deutschen Personals war, die tansanische Fachkräfte davon abhielt, sich bei der NECO zu bewerben. Es ist jedoch davon auszugehen, dass die besseren Verdienstmöglichkeiten in der Privatwirtschaft (oder im Ausland) eine größere Rolle spielten als dieser Faktor, denn selbst Counterparts, die die Aussicht hatten, Stellen von BRD-Experten bald zu übernehmen, verließen die NECO. Hinzu kamen Prestigefragen und mit dem Ingenieurberuf verbundene Statusvorstellungen: In einem Fall wurde ein junger tansanischer Bauingenieur von den Arbeitern nicht akzeptiert, „weil er keine Erfahrung hat und nicht selbst mit zufassen will“; andererseits weigerte er sich, in die Montageüberwachung zu gehen, weil er in dieser Tätigkeit keinerlei Nutzen sah. Die ideologischen Rivalitäten des Kalten Krieges waren angesichts der Personallage gänzlich bedeutungslos; das westdeutsche Team bemühte sich etwa intensiv um einen Tansanier, der nach sieben Jahren seinen Studienabschluss in der Sowjetunion erhalten sollte und schickte einen Mitarbeiter für eine fünfmonatige Fortbildung in die UdSSR. ${ }^{90}$

\section{Breite Allianzen für ein „deutsches Management“}

Die BMZ-Beamten wagten einen Kreuzzug für ihre entwicklungspolitischen Prinzipien. Die Rationalität Technischer Hilfe, erinnerten sie gebetsmühlenartig, beruhe auf dem Prinzip der Wissensvermittlung. Ohne Adressaten sei dieses Prinzip nicht umsetzbar. Zudem laufe die GTZ (und damit die Bundesregierung) Gefahr, sich durch die Stellung einer „Führungsmannschaft“ für ein Unternehmen der Wettbewerbsverzerrung schuldig zu machen - was für das BMZ ein ernsthaftes „ordnungspolitisches Problem“ darstellte. ${ }^{91}$ Mit ihrem Pochen auf Prinzipien konnten sich die BMZ-Beamten gegen Interessen der vielen anderen Akteure, die sich hier in einer unwahrscheinlichen Allianz verbündeten, jedoch nicht durchsetzen, was einmal mehr die Schwäche entwicklungspolitischer Ak-

89 Sämtliche Zitate aus BArch Koblenz, B 213/33089, Wiegmann (BMZ) an Sinn (GTZ), o.O., 4.11. 1980. Informationen zur Nachverfolgung der Vorwürfe finden sich in ebd., Merz/Rinne (GTZ) an Wiegmann (BMZ), Eschborn, 29.1.1981; ebd., Wiegmann (BMZ) an GTZ, Bonn, 9. 2.1981.

90 Afrika Bureau Cologne e.v., 4. Quartalsbericht 1.2.1976-30.4.1976 über die Tätigkeit des Beraters H.-G. T. für die GTZ, Köln, o.D., S. 17-18.

91 BArch Koblenz, B 213/33089, Wiegmann (BMZ) an Minister Offergeld, Bonn, 30.6.1981; ebd., B 213/33089, Wüstermann (BMZ) an Referat 114, Bonn, 14.9.1978. 
teure im Ringen mit gegensätzlichen politischen und wirtschaftlichen Interessen zeigte. Angesichts des breiten Widerstandes und des möglichen „Flurschaden[s]“ - politisch für die BRD, wirtschaftlich für Tansania, wie es in einem Schreiben hieß - gaben die BMZ-Beamten schließlich nach und stimmten der Verlängerung des Projekts unter dem statussichernden Etikett der Technischen Hilfe zu. ${ }^{92}$ Entwicklungspolitische Kriterien (wie z. B. die Ausbildungsfunktion der NECO durch die Bereitstellung von Praktikumsplätzen für Bildungsinstitutionen) blieben für diese Entscheidung annähernd bedeutungslos. Die Beamten im BMZ waren mit ihrer Position isoliert und standen einer breiten Allianz gegenüber.

NDC-Leiter Kilewo und die deutschen Experten hatten eine strategische Gruppe gebildet, unterstützt von der GTZ-Zentrale. Den Ausschlag im Tauziehen zwischen Technischer und Finanzieller Hilfe gab schließlich der Meinungswandel der Botschaft, die das BMZ erst auf die Probleme aufmerksam gemacht hatte, dann aber auf Deils Argumentationslinie umgeschwenkt war. Der Umschwung in der Botschaft resultierte aus der kontinuierlichen Bearbeitung durch die tansanischen Führungskräfte der NDC und den Staatssekretär im Industrieministerium, der für die NDC zuständig war. Wie war aber die tansanische Führungsriege zu der Meinung gekommen, dass ein direktes Verhältnis zwischen den Experten und der NECO „die Effizienz der deutschen ,Beratung' schmälern“ würde und ein „Minimum an Durchsetzungsvermögen gegenüber der tansanischen Führung (in der NDC vor allem) nicht mehr gewährleistet“ wäre? ${ }^{93}$ Wieso teilten NDC-Leiter Kilewo und Staatssekretär Mujuni nicht die Meinung der BMZ-Beamten, dass die NDC anstelle der GTZ die Verantwortung für die NECO übernehmen sollte?

Dass Deil und die anderen deutschen Experten die Akteure auf tansanischer Seite einfach überredeten und $\mathrm{zu}$ einem Meinungswechsel veranlassten, ist unwahrscheinlich. Es gab durchaus gute Gründe für die Tansanier, unter den gegebenen Bedingungen die Kontrolle abzugeben, selbst wenn das - zumindest kurzfristig - allen Nationalisierungsbestrebungen entgegenstand. Die Importsubstitution war um 1980 finanz- und wirtschaftspolitische Priorität und galt als geradezu überlebensnotwendig. Mittel- und langfristige Ziele zu verfolgen, wäre in dieser Situation nicht nur strategisch unklug, sondern realpolitisch unmöglich gewesen. Für die NDC-Leitung war der massive BRD-Personaleinsatz eine vergleichsweise „bequeme Lösung“ für das Problem der fehlenden tansanischen Fachkräfte, die ab einem bestimmten Qualifikationsgrad in die Privatwirtschaft abwanderten. ${ }^{94}$ Angesichts der politisch um 1980/81 noch unverrückbaren Posi-

92 Ebd., B 213/33100, Wiegmann (BMZ) an Referat 122, Bonn, 11.9.1981, S. 3.

93 Ebd., B 213/33089, Kremer (BRD-Botschaft) an AA, Dar es Salaam, 19.6.1981.

94 Ebd., B 213/33089, Wiegmann (BMZ) an Minister Offergeld, Bonn, 30.6.1981. 
tion, was Gehaltsanhebungen und Leistungsboni im öffentlichen Sektor betraf, hatten die Funktionäre hier keinerlei Spielraum. Hinzu kamen die drohenden wirtschaftlichen Schäden, die durch die angedrohten Rücktritte von den Aufträgen entstanden wären, was die NECO in eine Krise gestürzt hätte, die sich auch auf die Belegschaft ausgewirkt hätte. Demzufolge waren auch Partei- und Gewerkschaftsvertreter hier für die fortgesetzte Präsenz der Westdeutschen, zumal auf den unteren Ebenen leistungsbezogene Löhne und Bonuszahlungen eingeführt werden konnten und, im Gegensatz zu anderen sanierungsbedürftigen Betrieben die Angestelltenzahlen in der NECO seit Projektbeginn um 40\% gestiegen waren und die NECO als einer der wenigen NDC-Betriebe Gewinne abwarf. Zwar ging die Leitung auf manche Forderungen der Arbeiterschaft, wie etwa die Bereitstellung eines eigenen Firmentransports, nicht ein; Belege für größere Proteste oder anhaltende Missstimmungen sind aber nicht überliefert. Hinzu kamen biografische Faktoren: Staatssekretär Mujuni hatte mit einem DAAD-Stipendium Volkswirtschaftslehre in der BRD studiert, auf eigene Kosten ein Doktoratsstudium in Erlangen angehängt und nach seiner Rückkehr 1972 engen Kontakt zu westdeutschen Institutionen aufrechterhalten. ${ }^{95}$ In seiner Funktion im Industrieministerium setzte Mujuni auf gegenseitig vorteilhafte Verbindungen mit westlichen Firmen.

Das Projekt wurde unter der Bedingung, dass Tansania bald Counterparts stellte und die Verantwortung für die NECO übernahm, verlängert und lief noch bis in die 1990er Jahre. Der Managementwechsel fand bereits Mitte der 1980er Jahre statt, und 1987 kam die Botschaft zu dem Schluss, dass NECO zu den Projekten gehörte, bei denen der Wechsel des Managements in tansanische Hände „sogar zu Verbesserungen des Leistungsstandards geführt“ hatte. ${ }^{96}$ Als besonderer Erfolg galt, dass es NECO gelungen war - entgegen den tansanischen Bestimmungen, wie die Botschaft betonte - einen leistungsbezogenen Lohn einzuführen. ${ }^{97}$

95 DAAD-Archiv, B 212/91392/4, Stephan (DAAD), Handschriftlicher Vermerk über Gespräch mit Dr. Mujuni (Director for Industrial Operations, Ministry of Industries), Dar es Salaam, 1.6.1978. 96 BMZ, B 213/63790, BRD-Botschaft Dar es Salaam, Entwicklungspolitischer Zweijahresbericht Tansania, Dar es Salaam, August 1987, S. 12.

97 DAAD-Archiv, B 212/91395/1, KAS (Bereich IIS-Auslandsinformation), Erfolge und Grenzen deutscher Entwicklungspolitik (Teil 2), 15.6.1984, S. 12-13. 


\subsection{Die letzten Sozialisten: DDR-Planungsberater auf dem Festland (1981-1989)}

Die drei DDR-Planungsberater, die zwischen 1981 und 1989 in Tansania im Einsatz waren, agierten an einer zentralen Konfliktlinie der 1980er-Jahre: Wie sollte das neue Tansania aussehen, an welchen Aspekten des Sozialismusprojekts sollte festgehalten werden - und war nicht vielleicht eine verstärkte zentrale Planung der richtige Ansatz, um der Wirtschaftsmisere Herr zu werden? Wie in Kapitel 3 geschildert, ist der Einsatz der Planungsberater im Kontext der tansanischen Wirtschaftskrise und der zunehmenden Abkehr westlicher Geber zu sehen, nachdem Nyerere 1979 die IWF-Delegation des Landes verwiesen hatte. Die politischen Rahmenbedingungen für den Einsatz waren also keinesfalls optimal, aber auch nicht gänzlich aussichtslos. Teile der Parteiführung und Regierung standen hinter der neuen Diversifizierungsstrategie und eine mal größere, mal kleinere Gruppe im Staatsapparat war einer Orientierung gen Osteuropa und womöglich gar einem Experimentieren mit der Planwirtschaft nicht abgeneigt. Die bereits im Gefolge der Deklaration von Arusha etablierte Planungskommission, die vom Präsidenten geleitet wurde, spielte nach wie vor eine wichtige Rolle in der Ressourcenallokation und Preisfestsetzung, übte jedoch nicht annähernd so einen zentralen Einfluss auf die Wirtschaftstätigkeit aus wie die Planungsapparate in kommunistischen Ländern. ${ }^{98}$ Ab 1986 beugte sich Tansania - selektiv den Strukturanpassungsforderungen von IWF und Weltbank, nachdem vorherige Reformen die makroökonomische Schieflage nicht hatten beheben können. Das verkürzte jegliche Planung auf kurzfristige Maßnahmen und hatte Auswirkungen auf das Entwicklungspersonal: 1986 bekam der ungarische Finanzberater seine Kündigung ausgehändigt. 1987 wurde die Position des DDR-Finanzberaters im Zusammenhang mit einem IWF-Vertragsabschluss nicht mehr verlängert. Damit war, berichtete der DDR-Experte im Planungsministerium, seine „Beraterfunktion die letzte in einer zentralen Wirtschafts- oder Finanzinstitution Tansanias, die von einem Vertreter eines sozialistischen Landes besetzt “ war. ${ }^{99}$ Die vereinzelten DDRExperten, die isoliert in verschiedenen Bereichen (Finanz-, Planungs-, Bauministerium) eingesetzt wurden, hatten es nach Meinung der Parteileitung mit „ausgesprochen komplizierten Verhältnissen, ja teilweise mit ablehnend, wenn

98 Robert J. Utz, Coordination of Economic Policy Formation and Implementation, in: Robert J. Utz, Hg., Sustaining and Sharing Economic Growth in Tanzania, Washington D.C. 2008, S. 303312, hier: S. 304.

99 BArch Berlin, DE 1/58114, R., Abschlußbericht über Auslandseinsatz, Berlin, 2.2.1988. 
nicht sogar feindlich gegenüberstehenden Partnern“ $\mathrm{zu}$ tun. ${ }^{100}$ Es mag daher überraschend erscheinen, dass Tansania 1988 und 1989 konkrete Schritte zur Bildung einer Planungskommission unternahm - unter Mitwirkung, aber in inhaltlicher Hinsicht gleichzeitig weitgehend unter Ausschluss des letzten DDRPlanungsberaters.

Der folgende Blick auf die Planungsberater und die Auseinandersetzungen, die sie führten, fügt dem Bild einer stufenweisen Abkehr vom Sozialismus ab etwa 1975 und der Neoliberalisierung nach 1985 eine Nuance hinzu. Allen DDR-Planungsberatern wurde bei Ankunft Improvisationsvermögen abverlangt, da ihre Funktion unklar war und sie nicht genau wussten, was erwartet wurde oder wie sie es umsetzen sollten. Die wichtigste Orientierung bot einerseits die vor dem Einsatz bilateral ausgehandelte Direktive, andererseits die Interessenlage der tansanischen Vorgesetzten. Die drei DDR-Planungsberater nahmen teilweise die gleichen Aufgaben wie ihre Vorgänger in Sansibar in den 1960er-Jahren wahr. Auch mit ihren westdeutschen Pendants in den staatlichen Betrieben und Finanzinstitutionen gab es Überschneidungen: Sie analysierten Investitionsvorhaben, prüften, ob die Material- und Personalvoraussetzungen erfüllbar waren (viele vorgeschlagene Industrieprojekte setzten z. B. den Import von Rohstoffen voraus) und gaben auf dieser Grundlage Entscheidungsempfehlungen ab. Gelegentlich besuchten sie Projektstätten, darunter auch die problembehaftete Textilfabrik in Mbeya, die in kommerzieller Zusammenarbeit mit Betrieben aus der DDR, Polen und der BRD aufgebaut wurde (siehe Kapitel 3). Im Kontrast zu den westdeutschen Wirtschaftsberatern, die mit selbstbewusstem Habitus von der universellen Gültigkeit ihrer stark betriebswirtschaftlich geprägten Logik und Arbeitsweise ausgingen, waren sich die DDR-Planungsberater über die Bewertungsmaßstäbe oft im Unklaren. Offensichtlich gaben weder der offizielle Marxismus-Leninismus noch das Planungswissen aus der DDR einen ausreichenden Rahmen, sodass die Berater zwischen projekt- oder unternehmensbasiertem Rentabilitätsdenken und politischen Kriterien schwankten. Aus ungeklärten Umständen umfassen die Unterlagen der DDR-Planungskommission nur die Korrespondenzen mit den letzten beiden, nicht aber mit dem ersten Berater, mit dem jedoch ein Interview geführt werden konnte. Die folgende Darstellung basiert dementsprechend auf heterogenen Quellen.

100 SAPMO BArch Berlin, DY 30/15205, Bericht der Leitung an die Wahlberichtsversammlung der GO der SED DSM, Dar es Salaam, 25.11.1985, Bl. 166. 


\section{Planungswissen auf Anfrage (1981-1985)}

Ab den 1970er-Jahren erhielten DDR-Regierungsberater eine ausführliche fachliche und sprachliche Vorbereitung (siehe Kapitel 5); sie waren für den Einsatz also besser gewappnet als die meisten ihrer Vorgänger in Sansibar - was ihnen die mühsame Orientierung vor Ort aber nicht ersparte. $\mathrm{Zu}$ den wichtigsten Aufgabenbereichen des ersten Beraters gehörte, dem zuständigen Staatssekretär bzw. dem Abteilungsleiter durch Stellungnahmen und Analysen zuzuarbeiten:

Dann kam er hoch zu mir ins Zimmer, klatschte mir da was auf den Tisch und sagte, „Können Sie sich das mal angucken hier?“ Hat demnächst ein Gespräch mit Finanzgeber, Weltbank oder was, und möchte dazu mal eine Meinung haben. Oder der kam hier und sagte, „Können Sie mal dazu was sagen?“ So lief das ab. [...] Ist natürlich, wenn Sie das erste Mal so was machen, ist nicht so einfach, sage ich jetzt mal. [...] [M]an weiß ja nie, was die erwarten. Das war das erste. Die sagen dir ja auch nicht, was sie erwarten. Also mir haben sie‘s zumindest nicht gesagt. Die haben gesagt, die möchten meine Meinung dazu hören. Wie eine Meinung sein soll oder kann, wie sie sich das vorstellen, was sie unter Meinung verstehen, das wusste ich alles nicht. ${ }^{101}$

Der Berater bekam keine expliziten Rückmeldungen auf seine Arbeiten, vermutete aber - nicht zuletzt aufgrund der angebotenen Vertragsverlängerung - dass seine Analyseergebnisse in Verhandlungen mit Gebern verwendet wurden. Ein zweiter Arbeitsbereich war die Förderung des Planungswesens. Im Interview stellte der ehemalige Berater - womöglich verstärkt durch den Wunsch, sich von „Propagandisten“ abzugrenzen - die Vermittlung des DDR-Planungsmodells nur teils der Direktive, mehr aber noch der tansanischen Nachfrage entsprechend dar. Der Planungsberater hielt Vorträge über das DDR-Planungssystem, erarbeitete verschiedene Diagramme und Skripten zu Einzelaspekten der Planung (Territorialplanung, Bauplanung, Arbeitskräfteplanung, Schulplanung) und organisierte Weiterbildungskurse für Regionalplaner. Von Besserwisserei oder überhaupt einem Gefühl des Wissensvorsprungs, vor allem gegenüber den tansanischen KollegInnen im Ministerium, konnte dabei aber keine Rede sein, betonte er:

Ich habe denen ja nichts beigebracht, also was soll ich denen beibringen, die waren teilweise besser ausgebildet als ich. Die waren studierte Ökonomen [...], haben in den USA studiert [...]. Das waren alles kluge Leute, mit denen konnte man sich auch entsprechend unterhalten. Die wollten in diese Richtung einfach wissen von mir, wie funktioniert dieses System hier, dieses Wirtschaftssystem und Planungssystem. Das wollten die wissen. Nicht, dass die das dann kopieren wollten, dazu war auch die politische Situation gar nicht da, die waren ja

101 Interview \#21, DDR-Planungsberater. 
viel zu viel abhängig von Weltbank, USA, Währungsfonds usw., also das wäre gar nicht gegangen. ${ }^{102}$

Wie in Kapitel 2 angesprochen suchten tansanische Akteure nicht nur in sozialistischen Ländern in Europa, sondern weltweit nach wirtschaftspolitischen Inspirationen, um Lösungswege für die ökonomische Krise zu finden. Als Alternativvorschlag, nicht als transferierbares Modell fasste dann auch der Berater die DDR-Planung auf, die nicht ohne Weiteres in Tansania eingeführt werden konnte. $\mathrm{Zu}$ dieser Meinung gelangte er nicht zuletzt, weil sein Zugang zu Informationen stets beschränkt blieb:

Die Tansanier haben ja auch ein ganz anderes System der Planung gehabt. Die können nicht sagen, „Das übernehmen wir jetzt und machen das so“, so geht’s ja nicht. Das ist ja mehr oder weniger mal eine Alternative, die man denen sagt - so könnte man's auch machen. Oder so machen wir es, oder versuchen wir es zu machen, guckt euch mal an, was man davon bei euch gebrauchen kann, denn ich habe das System, was die, oder wie die dort planen im Einzelnen ja nie so mitgemacht, da haben sie mich ja gar nicht reingucken lassen.

Die gleichmütige Darstellung des ersten Beraters im Interview steht in Kontrast zu dem angestrengten Ton in den Berichten des zweitens Beraters, in denen die kraftraubenden Anstrengungen um Einflussgewinn geradezu spür werden. Der Kontrast resultiert womöglich aufgrund verschiedener persönlicher Einstellungen, kann aber auch Resultat der anderen Sprecherposition sein. Während sich der erste Berater aus der Distanz erinnerte und keinerlei Nähe zu damaligen Problemen, Haltungen und Sprachregelungen mehr hatte, befand sich der zweite beim Schreiben seiner Berichte inmitten eines eng abgesteckten Handlungsfeldes, in dem er seine eigenen Qualitäten und Bemühungen genauso wie die Widrigkeit des Umfeldes betonte.

\section{Als Einzelkämpfer gegen den neoliberalen Trend (1985-1988)}

An die Vorarbeiten des Vorgängers anzuknüpfen war schwierig für den zweiten DDR-Planungsberater, denn kurz nach Ankunft des zweiten Planungsberaters wurden Planungs- und Finanzministerium unter der Leitung von Cleopa Msuya (der in anderen DDR-Berichten als „eine rechte Kraft“ identifiziert wurde ${ }^{103}$ )

102 Ebd.

103 BArch Berlin, DE 1/57846, R., „Halbjahresbericht für den Zeitraum 22.11.1987-31.5.1988“, Dar es Salaam, 8.6.1988, S. 1. 
zusammengelegt. Erst 1980 waren die beiden Ressorts aufgrund von Abstimmungsproblemen getrennt worden, die erneute Zusammenlegung ließ erwarten, dass die Konflikte wieder aufkeimen würden. Das Vertrauen in staatliche Planung war erschüttert, die präsidentielle Planungskommission verlor im Rahmen der Strukturanpassung mehr und mehr institutionelle Kompetenzen an das Ministerium. ${ }^{104}$ Der bisherige Planungsminister Malima, die treibende Kraft hinter der Planungskooperation, wurde auf eine „Juniorministerstelle“ im President's Office versetzt. Aus dem geplanten Einsatz als Berater beim stellvertretenden Staatssekretär wurde somit nichts. Andere leitende Beamte und Funktionäre, die bereits vorher mit dem DDR-Berater gut zusammengearbeitet hatten, blieben aber und boten Anknüpfungspunkte.

Trotz seiner schwierigen Ausgangsposition erkämpfte sich der zweite DDRPlanungsberater Zugang zu Entscheidungsgremien und war damit, soweit die Quellen diesen Einblick erlauben, umfassender informiert als noch sein Vorgänger. Bei einem Seminar zur Vorbereitung des nächsten Fünfjahresplans, an dem 25 Entscheidungsträger aus höchsten Ministeriums-, Partei- und Wirtschaftskreisen teilnahmen, war er der einzige Ausländer. Während diese Tatsache von anderen Entsandten als Zeichen des besonderen Einflusses oder der besonderen Integrationsleistung hervorgehoben worden wäre ${ }^{105}$, erschien sie bei ihm als Beleg für eine Außenseiterposition. Die Isolation spürte er auch sprachlich, da der Richtlinienentwurf nur auf Swahili vorlag (dessen er nicht mächtig war, wodurch ihm nicht nur wichtige Informationsquellen verschlossen und Dokumente vorenthalten blieben, sondern - wie er fand - ihm auch weniger Vertrauen entgegengebracht wurde und er in seinem „Wirkungsfeld [...] wesentlich eingeschränkt" war"106).

Mit seiner wirtschaftspolitischen Position stand er ebenfalls auf verlorenem Posten. Mit Besorgnis musste er hören, dass sich mehrere Seminarteilnehmer auf den „internationalen Trend“ der „Denationalisierung“ beriefen, der sich bereits in Großbritannien, China und der UdSSR niederschlug. Die Kontrolle wirtschaftlicher Schlüsselzweige durch die Regierung aufrechtzuerhalten, so meinten sie, bedeute nicht, dass diese Branchen im Staatseigentum verbleiben mussten. Selbst die CCM-Vertreter, in der Regel die stärksten Befürworter staatlicher Kontrolle, bauten keine Gegenposition auf: „So war ich der einzige Teilnehmer, der über den Inhalt der Maßnahmen zur Stärkung privater Initiative in der UdSSR und über die Hintergründe der Reprivatisierung in Großbritannien gesprochen und auf die

104 Utz, Coordination, S. 304.

105 Vgl. etwa Interview \#101, GTZ-Projektleiter.

106 BArch Berlin, DE 1/58114, R., Abschlußbericht über Auslandseinsatz, Berlin, 2.2.1988, S. 3; ebd., H.-P. R., Bericht - Berichtszeitraum 1.11.-31.12.1985, o.D. [1986], Dar es Salaam, S. 12. 
Notwendigkeit einer zentralen Wirtschaftsleitung und -planung hingewiesen hat. “107 Demgegenüber stand die Meinung, die Unternehmen sollten volle Autonomie erlangen, weil der Einfluss von Partei und Staat effektives Wirtschaften unmöglich mache. Das Vorhaben der „Denationalisierung“ stieß allerdings auf praktische und politische Probleme. Eins der größten war die Relation zum Weltmarkt: Ausländische Investoren waren nicht bereit, die maroden staatlichen Unternehmen aufzukaufen; zudem lagen die Profite bereits präsenter Firmen aufgrund der Devisenknappheit in Tansania auf Eis und konnten nicht ins Ausland transferiert werden, was potenzielle Investoren zusätzlich abschreckte. Gelder von westlichen Gebern kamen fast ausschließlich für Vorhaben, die an Abnahmeverpflichtungen gebunden waren, während sich für Industrieprojekte auf lokaler Rohstoffbasis keine Interessenten fanden. Auch innenpolitische Konfliktlinien brachen an der Frage von Privatisierung und Deregulierung wieder auf. Die größten Spannungen während des Seminars offenbarten sich dem Bericht des DDR-Beraters zufolge zwischen Teilnehmern afrikanischer und asiatischer Herkunft, da von den Ersteren einige die Herkunft als Hauptkriterium zur Förderung des Unternehmertums heranziehen wollten, um den Einfluss „indischer“ Netzwerke und Firmen zu reduzieren. Der ethnische Faktor im Kampf um die Früchte der Privatisierung sollte ein Kernthema politischer Debatten Anfang der 1990er-Jahre werden. ${ }^{108}$ Der DDR-Berater fügte im Bericht hinzu, dass der Wirtschaftssektor nicht nur von „Indern“, sondern auf afrikanischer Seite auch von Angehörigen der Chagga-Ethnie dominiert werde. Mit der Verlautbarung einer eigenen Position in diesen heiklen Fragen hielt er sich, wie schon seine Vorgänger auf Sansibar, allerdings zurück. Deutlich vernahm der Berater darüber hinaus den wachsenden Druck auf den leadership code von 1967, den einige Seminarteilnehmer abschaffen oder zumindest aufweichen wollten, um auch Partei- und Staatsfunktionären den Einstieg in die Geschäftswelt zu ermöglichen. Hier handelte es sich um jene Vertreter aus dem Staats- und Parteiapparat, die ihren politischen Einfluss in ökonomisches Kapital umwandeln wollten und dafür das Risiko eingingen, an den seit 1967 (zumindest auf der Ebene politischer Richtlinien) unberührbaren Prinzipien zu rütteln. ${ }^{109}$

Jenseits des Seminars war das Wissen des DDR-Beraters um Planungsmethoden durchaus nachgefragt und fand in den hohen Ministeriumsrängen

107 Ebd., R., Informationen über ein Seminar zur Vorbereitung des 2. Fünfjahresplanes der Vereinigten Republik Tansania, Dar es Salaam, 15.4.1987.

$108 \mathrm{Zu}$ den Beziehungen zwischen der afrikanischen Bourgeoisie und indisch-tansanischer Wirtschaftsmacht siehe Aminzade, Race, Kap. 9-10.

109 BArch Berlin, DE 1/58114, R., Abschlußbericht über Auslandseinsatz, Berlin, 2.2.1988, S. 10, 17. 
Anklang. Einige seiner Entwürfe wurden ans CCM-Zentralkomitee weitergeleitet und sein Vorschlag, die übliche Budgetplanung auf rein finanzieller Basis durch eine Materialplanung (Verfügbarkeit von Rohstoffen etc.) zu ergänzen, stieß sogar auf das Wohlwollen einiger Regierungsmitglieder. ${ }^{110}$ Auf seine Vorschläge hin wurden einige „Experimente zu einer zentralen Produktionsplanung“ in privaten und staatlichen Betrieben durchgeführt, die Einführung einer genuinen zentralisierten Planung scheiterte aber am Widerstand anderer Ministerien. ${ }^{111}$

Das Festhalten des zweiten Beraters an der planwirtschaftlichen Orientierung, das ihn in politischer Hinsicht isolierte, war einerseits der DDR-Direktive geschuldet, an die er sich halten musste; andererseits mag es auch damit zusammenhängen, dass er sich an das Bekannte klammerte, um seinen Kompetenzbereich nicht zu überschreiten. Wie er in seinem Abschlussbericht kritisierte, war er auf die Funktionsweise des kapitalistischen Wirtschafts- und Finanzwesens nicht genügend vorbereitet worden. ${ }^{112}$ Die von Tansania angebotene Vertragsverlängerung um weitere drei bis vier Jahre lehnte er mit Verweis auf den schlechten Gesundheitszustand seiner Frau ab.

\section{Plan-loser Außenhandel (1988-1989)}

Der dritte Berater hatte ebenfalls einen schweren Anlauf: Zur Einarbeitung durch seinen Vorgänger blieben nur drei Tage, die Kontaktaufnahme zu „eigentlichen Auftraggebern“ war unmöglich, ein Gespräch mit dem neuen Minister Mbogoro kam nie zustande: „Die Absicht, mich von vornherein als Berater dem Staatsminister zuzuordnen, [konnte] nicht realisiert werden“, womit er sich ohne „Plan oder Programm“ im institutionellen Niemandsland befand. Die Bemühungen des zweiten Beraters, neben der Finanz- auch die Materialplanung und weitere in der DDR übliche Planungstechniken einzuführen, verpufften wirkungslos oder verschlissen „im Getriebe des Apparates“:

Trotz intensiver Bemühung gelang es nicht, die bereits im Frühjahr 1987 von der Regierung bestätigten Kennziffern in die Richtlinien für die Erarbeitung des Planes 1988/89 aufzunehmen. Es wurde Zeitmangel als Grund vorgeschoben. In Wahrheit gibt es jedoch nach wie vor keine Bereitschaft, die Planung auf materielle Kennziffern auszudehnen. ${ }^{113}$

110 Ebd., R., Halbjahresbericht - Berichtszeitraum II. Halbjahr 1986, Dar es Salaam, 2.1.1987.

111 Ebd., R., Abschlußbericht über Auslandseinsatz, Berlin, 2.2.1988, S. 10, 17.

112 BArch Berlin, DE 1/58114, R., Abschlußbericht über Auslandseinsatz, Berlin, 2.2.1988, S 2. 113 BArch Berlin, DE 1/57846, R., Halbjahresbericht für den Zeitraum 22.11.1987-31.5.1988, Dar es Salaam, 8.6.1988, S. 9. 
Nach wie vor gab es kein Organ, das die Planerfüllung kontrollierte oder eine gemeinsame Planung mit Sansibar anstrebte. Nach sieben Jahren war also nur eine „geringe Effektivität der Beratereinsätze“ zu konstatieren; die Einführung einer Planwirtschaft war nicht wirklich vorangekommen: „Das ist aber nicht die Schuld der eingesetzten DDR-Berater, die mit viel Fleiß und Initiative tätig waren und deren Einsatz anerkannt wird, sondern ist der politischen Gesamtkonstellation zuzuschreiben“, meinte er schon kurz nach seiner Ankunft. ${ }^{114}$ Im Gegensatz zu seinem Vorgänger bewies er einen größeren Anpassungswillen an diese „Gesamtkonstellation“, zumal er „[n]eben einer Vielzahl von Beratern aus kapitalistischen Ländern und internationalen Organisationen“, die jetzt wieder verstärkt ins Land kamen, nunmehr „der einzige Vertreter eines sozialistischen Landes [...] im gesamten zentralen Staatsapparat“ war. ${ }^{115}$

In mancher Hinsicht waren die Voraussetzungen zur Einführung der zentralen Planung aber wieder günstiger als noch für seinen Vorgänger: 1988 bäumten sich die AnhängerInnen von Ujamaa noch einmal auf. Das neu verabschiedete Parteiprogramm war ein klares Bekenntnis zum Sozialismus, die Liberalisierungspolitik wurde vom Parteivorsitzenden Nyerere unter Beschuss genommen, anstelle von „Denationalisierung“ ging der Trend wieder in Richtung Nationalisierung. Der von Finanz- und Planungsminister Msuya vorgelegte Fünfjahresplan wurde im Parlament mit dem Argument zurückgewiesen, dass er den Parteirichtlinien nicht genügend entspreche; Msuya verlor zudem seinen Posten im Politbüro.

Sein Hauptaugenmerk legte der dritte DDR-Planungsberater auf zwei Aktivitäten. Erstens lotete er, da er selbst Außenhandelsspezialist war, Handels- und kommerzielle Entsendungsmöglichkeiten für die DDR aus und erarbeitete auf tansanische Anfrage Exportstrategien, entfernte sich also teilweise von Planungsfragen im engeren Sinne; zweitens bemühte er sich, bei den Vorbereitungen zur Bildung einer nationalen Plankommission einen Fuß in die Tür zu bekommen, um dem DDR-Planungsmodell dort mehr Einfluss zu verschaffen. Trotz größerer Vorerfahrungen im Außenhandel kritisierte er wie sein Vorgänger die ungenügende Vorbereitung in der DDR, da er das „tiefere Eindringen in den kapitalistischen Wirtschafts- und Finanzmechanismus [als] unerläßlich“ erachtete. ${ }^{116}$

Zur Unterstützung der DDR-Außenwirtschaft sandte der Berater ausführliche Analysen über die politische und wirtschaftliche Situation Tansanias in die DDR, knüpfte Kontakte mit der Bank von Tansania und dem Büro für Außenhandel,

114 Ebd., S. 17.

115 Ebd.

116 Ebd., S. 15. 
besuchte Messen und erarbeitete konkrete Vorschläge, die dann direkt von der Plankommission an das Ministerium für Außenhandel weitergeleitet wurden. Die direkte Einbindung in den Außenhandel war also bei den Planungsberatern mehr als bei allen anderen Auslandskadern gegeben. Ost-Berlin teilte er sein eindeutiges Urteil mit, „daß jede Neugewährung von Krediten falsch wäre, da es bei der gegenwärtigen Politik faktisch keine Möglichkeit der Rückzahlung gibt“, womit er zur Vorsicht der DDR beitrug, keine weiteren Großprojekte in Tansania anzugehen. ${ }^{117}$ Durch seine guten Kontakte identifizierte er jedoch kleinere Geschäftsmöglichkeiten, z. B. die Lieferung von Chemikalien an Firmen, die autonom über ihre Devisenvorräte entscheiden durften. Zumindest im Hinblick auf die Rückzahlung der Kredite hatte auch die DDR ein Interesse, dass Tansania das aufoktroyierte makroökonomische Sanierungsprogramm durchführte.

Die Berichte des dritten Beraters enthielten ähnlich den Korrespondenzen in der wirtschaftlich orientierten Mittag-Kommission kaum marxistisches Vokabular. Der Ton zeichnete sich durch eine betonte Nüchternheit aus, die ihre inhaltliche Entsprechung in einem Einlenken gegenüber westlichen Forderungen fand. Gegen die Privatisierungswelle und den Abbau sozialer Leistungen anzugehen hielt er für aussichtslos, da „gegenwärtig auch keine reale Alternative zu dieser Politik“ existiere. ${ }^{118}$ Im Unterschied zu seinem Vorgänger (aber ähnlich wie DDR-Planungsberater in den 1960er-Jahren in Sansibar) hatte er in seiner Arbeit „stets versucht, deutlich zu machen, daß private Initiative und ausländische Kapitalbeteiligung nützlich für die Entwicklung der Volkswirtschaft sein können, wenn das von der Partei gestellte Ziel der Errichtung einer sozialistischen Gesellschaft nicht aus dem Auge verloren wird“. ${ }^{119}$ Seine Analysen orientierten sich nicht an sozialen Kriterien wie Gleichheit oder einer Unterstützung des Sozialismus, sondern - ebenso wie die Analysen westlicher Technokraten - an nüchternen volkswirtschaftlichen Kriterien. So kritisierte er die egalitäre Lohnpolitik als „zunehmende Gleichmacherei“, da sich die „Verringerung der Differenzen zwischen niedrigen und hohen Einkommen negativ auf Leistungsentwicklung und

117 BArch Berlin, DE 1/57846, R., „Information über die von der Weltbank vorgenommene Einschätzung der Entwicklung der Zahlungsbilanz Tansanias in den Jahren 1989-1999“, Dar es Salaam, 5. Mai 1989, S. 4; ebd., H. R., Analyse des Außenhandels der Vereinigten Republik Tansania, Dar es Salaam, 17.4.1988, S. 14.

118 Ebd., R., Halbjahresbericht für den Zeitraum Januar bis Juni 1989, Dar es Salaam, 20.6.1989, S. 1.

119 BArch Berlin, DE 1/57846, R. an Stv. SPK-Vorsitzenden Albrecht, Dar es Salaam, 15.3.1988, BArch Berlin, DE 1/57846, S. 4. 
Stimulierung“ auswirken und „zum Abgang qualifizierter Kader ins Ausland oder in die Privatwirtschaft" führen würden. ${ }^{120}$

Im Februar 1988 beschloss das CCM-Zentralkomitee die Bildung einer Plankommission. Über ein Jahr später, am 24.4.1989, verabschiedete das Parlament ein Gesetz zur Etablierung einer nationalen Plankommission. Weitere zwei Monate darauf hatte der DDR-Berater weder den Gesetzestext vorliegen noch den Stellenplan für die Kommission zu Gesicht bekommen. Sein Wunsch zur Beteiligung war mit dem Verweis auf fehlende Swahili-Kenntnisse immer wieder zurückgewiesen worden. Einzig durch die gelegentliche Einsicht in Grundsatzdokumente, zu denen er dann eigene Stellungnahmen erarbeitete, gelang es ihm, sich Zugang zu leitenden Funktionären zu verschaffen und seine Positionen einfließen zu lassen. Malima, der die Leitung der Kommission übernehmen sollte, gab ihm den Auftrag, ein Planungshandbuch zu erstellen. ${ }^{121}$ Die konstanten Bemühungen des dritten Beraters, Kontakte zu den einflussreichen Personen im Planungsbereich zu knüpfen, waren nicht gänzlich erfolglos; gebildete Arbeitsgemeinschaften zur Planungskommission stützten sich teilweise auf seine Ausarbeitungen, er selbst durfte allerdings nicht teilnehmen. ${ }^{122}$ Der Vertrag des Beraters lief im Januar 1990 aus, die archivalische Spur verliert sich hier.

Obwohl alle drei Berater den prestigereichen Rang als „Regierungsberater“ innehatten, blieben ihre Kontakte und ihr Einfluss weitgehend auf die mittlere Ebene beschränkt. Während vor allem der zweite Berater seinen Fokus stark auf die Einführung einer zentralen Wirtschaftsplanung richtete und damit auf Widerstand stieß, konzentrierte sich der letzte auf Außenhandelsfragen. Die Dekolonisierung der Wirtschaftsbeziehungen, wie sie noch in den 1960er Jahren in Sansibar ganz oben auf der Agenda gestanden hatte, wurde von ihm angesichts der ökonomischen Lage Tansanias und der daraus resultierenden Machtbeziehungen schon längst für eine Illusion gehalten.

\section{Fazit}

Die vier Fallstudien zeigen, dass die wirtschaftlichen Interessen des Entsendelandes die Beratungstätigkeit immer wieder beeinflussten - mal negativ und gegen den Willen der Berater, wie im postrevolutionären Sansibar, mal stark von den Beratern selbst forciert, wie besonders bei den westdeutschen Beratern, aber

120 Ebd., R., Halbjahresbericht für den Zeitraum Januar bis Juni 1989, Dar es Salaam, 20.6.1989, S. 5 .

121 Ebd., S. $10-12$.

122 Ebd., R. an Stv. SPK-Vorsitzenden Albrecht, Dar es Salaam, 19.9.1989. 
teilweise auch bei den DDR-Planungsberatern deutlich wurde. Die Berater waren - teils ungewollt, teils mit eigener Beteiligung - Komplizen der Wirtschaftsinteressen von Firmen (BRD) oder ihres Entsendestaates (DDR). Die kritische Beratungsfunktion wurde logischerweise immer dann ausgesetzt, wenn Beziehungen zu eigenen Wirtschaftsverbindungen in DDR und BRD (und womöglich darüber hinaus) auf dem Spiel standen.

Um praxiswirksam zu werden, waren die Berater auf Allianzen mit tansanischen Regierungs- und Verwaltungskreisen (weniger der Partei) angewiesen, wobei hier einmal mehr in Erinnerung zu rufen ist, dass diese Elite selbst nicht homogen war, die Berater also oft zwangsläufig Partei für eine Seite ergreifen mussten, mit ihr identifiziert wurden und dann von dieser abhängig waren. Waren die Berater weitgehend auf sich allein gestellt, konnten sie Angebote und Pläne kritisch unter Lupe nehmen, tansanischen Vorgesetzten wertvolle Argumente für Verhandlungen mit ausländischen Investoren liefern und ein Gegengewicht zu anderen Beratern darstellen. Je unabhängiger ein Berater war, desto prekärer war jedoch auch seine Situation unter ungünstigen Rahmenbedingungen.

Gleichzeitig hatten Sansibar und Tansania - zumindest zeitweise - auch ein Interesse daran, die Berater gleich „im Paket“ mit den Investitionsmöglichkeiten zu rekrutieren: So wie Karume sich mithilfe von DDR-Investitionen vom imperialistischen, kapitalistischen Westen abkoppeln wollte, profitierten die Funktionäre in der NDC von Geschäftskontakten und Warenhilfe, die mit dem GTZPersonal verbunden waren. Die Prädispositionen der deutschen Berater Richtung Markt- oder Planwirtschaft waren auf tansanischer Seite bekannt, sodass sich hier besonders enge Allianzen bildeten. Das politische Kapital der Berater, dass sie nämlich zur „sozialistischen Staatengemeinschaft“ gehörten, war der entscheidende Grund für Tansanier wie Karume oder Malima, um diese Einsätze zu fordern - allerdings in erster Linie in der Hoffnung auf weitere wirtschaftliche Investitionen, nicht auf den Transfer von DDR-Konzepten. Die planwirtschaftlichen Ansätze wurden im Tagesgeschäft zerrieben, was zum Teil am mangelnden Interesse der Counterparts, vor allem aber an fehlenden Voraussetzungen lag.

Der massive Personaleinsatz von GTZ-,,Beratern“ bei der NECO führte, entgegen den entwicklungspolitischen Prinzipien, zu einer neokolonialen Abhängigkeitsbeziehung, die nur vonseiten des BMZ (zumindest teilweise) problematisiert wurde, während alle anderen Akteure sich für ihre Aufrechterhaltung einsetzten. Das war kein Einzelfall, Vergleichbares geschah z.B. auch bei der National Transport Corporation. Solche „Übernahmen“ durch ein ganzes Dutzend von „Beratern“ konnten zur wirtschaftlichen Funktionsweise eines Betriebs beitragen, konsolidierten aber bestehende Abhängigkeitsverhältnisse und Ungleichheiten, statt zu ihrer Überwindung beizutragen. 
Ebenfalls sichtbar wurden verschiedene Obstruktionsstrategien, mit denen sich Berater auseinandersetzen mussten. Direkte Counterparts sowie politische Akteure und BeamtInnen sorgten dafür, dass sie aus Sitzungen und Gremien ausgeschlossen blieben, Weisungsbefugnisse verloren oder keine Vertragsverlängerungen erhielten. Daniel Bendix hat entsprechende Vorgehensweisen in einem aktuelleren Fallbeispiel als Widerstand gegen „koloniale Macht“ gedeutet. ${ }^{123}$ Eine solche Interpretation lässt sich auf die dargestellten Beispiele kaum anwenden, denn oft ging es gleichzeitig um das Ausfechten interner Machtkämpfe oder um die Aufrechterhaltung von Ausbeutungsmechanismen. Der Widerstand gegen ausländische Berater gründete etwa im Falle des Edelsteinberaters darin, dass eine korrupte, internationale Allianz von Wirtschaftseliten sich gegen ihn wandte, weil er die für Tansania nachteiligen Abkommen kritisiert und sogar ein Prüfungsverfahren dagegen eingeleitet hatte.

Im Vergleich des Habitus von DDR- und BRD-Beratern fällt auf, dass die westdeutschen Berater selbstbewusster, bisweilen gar forsch und offensiv fordernd auftraten, während ihre ostdeutschen Pendants versuchten, ihrer Meinung auf subtilere Weise Geltung zu verschaffen. Ohne konkrete Aufträge fühlten letztere sich oft orientierungslos. Neben Faktoren wie Sprachsicherheit, internationaler Erfahrung und Glauben an den eigenen Einfluss spielten hier auch die Direktiven und Biografieerwartungen der DDR-Berater eine Rolle. Für sie war der Einsatz immer auch eine politische Tätigkeit zur Verbesserung der zwischenstaatlichen Beziehungen. Gerade aus diesem Grund waren sie aber auch besonders bemüht, die DDR-Leitlinien mit den Interessen hoch- und gleichrangiger Counterparts in Einklang zu bringen; in der Praxis waren sie keine dogmatischen Propagandisten des Marxismus-Leninismus, sondern manövrierten eklektizistisch zwischen den konkurrierenden Interessen verschiedener Akteure und Felder. Berater, die diese Manövriertechnik nicht beherrschten, waren einem sehr viel höheren Risiko ausgesetzt, vor Ort marginalisiert oder von Ost-Berlin abberufen zu werden. Während die DDR-Berater, viele von ihnen Beamte, stark an ihr Rollenverständnis als Vertreter des Staates gebunden waren, nutzen westdeutsche Berater als beruflich Selbstständige den Entsendestatus selektiv und strategisch. So nahm Ingenieur Deil den staatlich-entwicklungspolitischen Überbau gern in Anspruch, um damit das eigene Prestige zu erhöhen und sogar eine Oppositionsstellung gegen das BMZ zu riskieren, das ihn dezidiert nicht als Vertreter der BRD sah.

123 Daniel Bendix, Resistance or Damp Squibs? Challenges to Colonial Power in Contemporary German Development Interventions in the Area of Reproductive Health in Tanzania, in: Journal für Entwicklungspolitik XXX/1 (2014), S. 21-43. 
Der langfristige Anspruch, die internationalen Wirtschaftsbeziehungen grundlegend $\mathrm{zu}$ transformieren und $\mathrm{zu}$ „dekolonisieren“, war lediglich für die DDR-Berater auf Sansibar handlungsanleitend. Der Glaube, dass Politik die Wirtschafts- und Handelsstrukturen langfristig beeinflussen könnte, nahm sukzessive ab. Tansania forderte in den 1970er-Jahren westdeutsche Berater an, um staatliche Betriebe wirtschaftlich zu sanieren und bestenfalls sogar neue Investitionskanäle zu eröffnen. Für derartige Aufgaben waren technokratische westliche Consultants besser geeignet als Entsandte aus sozialistischen Staaten, deren Erfahrungshorizont in einer Planwirtschaft wenig Umgang mit Investoren und staatlichen Betrieben außerhalb ihres Heimatlandes ermöglicht hatte. Die DDRPlanungsberater in den 1980er-Jahren beklagten ihr Unwissen in Bezug auf kapitalistische Finanzmechanismen, die die Entwicklungspolitik zunehmend prägten, und sahen keinerlei Möglichkeit, wirtschaftliche Strukturen und Beziehungen grundlegend umzustrukturieren. De facto sollten sie die wirtschaftlichen Außenbeziehungen Tansanias (sowie der DDR selbst) möglichst kurzfristig optimieren - und hatten damit den Horizont einer langfristigen „Dekolonisierung“ von Finanzen und Handel, wie er noch die Tätigkeiten ihres Vorgängers Gentsch in Sansibars geleitet hatte, aus den Augen verloren. 